\title{
Evidence for radical fragmentations from persistent singlet carbenes
}

Xavier Cattoën, Karinne Miqueu, Heinz Gornitzka, Didier Bourissou, * Guy Bertrand*

\section{SUPPORTING INFORMATION}

\section{Synthesis and Spectroscopic Data}

All manipulations were performed under an inert atmosphere of argon using standard Schlenk techniques. Dry, oxygen-free solvents were employed. ${ }^{1} \mathrm{H}$ and ${ }^{13} \mathrm{C}$ NMR spectra were recorded on Bruker WM250, Avance 300, Varian Inova 300 or Inova 500 spectrometers. ${ }^{1} \mathrm{H}$ and ${ }^{13} \mathrm{C}$ chemical shifts are reported in ppm relative to $\mathrm{Me}_{4} \mathrm{Si}$ as external standard. N,Ndiisopropyl-chloroformamidinium chloride and $\mathrm{N}, \mathrm{N}$-dimethyl- $\mathrm{N}^{\prime}$-isopropylhydrazine were synthesized according to litterature procedures. ${ }^{\mathrm{S} 1, \mathrm{~S} 2}$

Synthesis of the 1-(isopropylamino)piperidine. 1-aminopiperidine (15 mmol) was refluxed in acetone $(100 \mathrm{~mL})$ over magnesium sulfate for 3 hours. The solution was filtered, then concentrated to afford the hydrazone (2.0 g, 96\%). ${ }^{1} \mathrm{H}$ NMR $\left(\mathrm{CDCl}_{3}\right): \delta 1.4\left(\mathrm{~m}, 2 \mathrm{H}, \mathrm{CH}_{2}\right)$, $1.6\left(\mathrm{~m}, 4 \mathrm{H}, \mathrm{CH}_{2}\right), 1.88$ [s, $\left.3 \mathrm{H}, \mathrm{NC}\left(\mathrm{CH}_{3}\right)_{2}\right], 1.92$ [s, $\left.3 \mathrm{H}, \mathrm{NC}\left(\mathrm{CH}_{3}\right)_{2}\right], 2.5\left(\mathrm{~m}, 4 \mathrm{H}, \mathrm{NCH}_{2}\right)$; ${ }^{13} \mathrm{C}\left\{{ }^{1} \mathrm{H}\right\}\left(\mathrm{CDCl}_{3}\right): 18.0\left[\mathrm{NC}\left(\mathrm{CH}_{3}\right)_{2}\right], 23.7\left(\mathrm{CH}_{2}\right), 24.9$ [NC( $\left.\left.\mathrm{CH}_{3}\right)_{2}\right], 25.2\left(\mathrm{CH}_{2}\right), 55.8\left(\mathrm{NCH}_{2}\right)$, $164.6\left[\mathrm{~N}=\mathrm{C}\left(\mathrm{CH}_{3}\right)_{2}\right]$.

The hydrazone (14 mmol) was then dissolved in acetonitrile $(50 \mathrm{~mL})$. Sodium cyanoborohydride $(0.60 \mathrm{~g}, 16 \mathrm{mmol})$ was then added in 5 portions over 30 minutes. After 45 minutes of stirring, glacial acetic acid $(2.7 \mathrm{~mL}, 43 \mathrm{mmol})$ was added. The mixture was stirred 6 hours, then treated with hydrochloric acid (37\%, $13 \mathrm{~mL})$. The solution was concentrated in vacuo. A $10 \%$ sodium hydroxide solution was then added until the solution was strongly basic. The hydrazine was then extracted with ether $(3 \times 50 \mathrm{~mL})$, and the solution concentrated, yielding the hydrazine (2.0 g, 98\%). ${ }^{1} \mathrm{H}$ NMR $\left(\mathrm{CDCl}_{3}\right): \delta 0.99\left[\mathrm{~d},{ }^{3} J_{\mathrm{HH}}=6.0 \mathrm{~Hz}, 6 \mathrm{H}\right.$, $\mathrm{NCH}\left(\mathrm{CH}_{3}\right)_{2}$ ], $1.4\left(\mathrm{~m}, 2 \mathrm{H}, \mathrm{CH}_{2}\right), 1.6\left(\mathrm{~m}, 4 \mathrm{H}, \mathrm{CH}_{2}\right), 2.5$ (m, $\left.4 \mathrm{H}, \mathrm{NCH}_{2}\right), 2.98$ [sept, ${ }^{3} J_{\mathrm{HH}}=$ 6.0 Hz, $\left.1 \mathrm{H}, \mathrm{NCH}\left(\mathrm{CH}_{3}\right)_{2}\right] ;{ }^{13} \mathrm{C}\left\{{ }^{1} \mathrm{H}\right\}\left(\mathrm{CDCl}_{3}\right): 22.0\left[\mathrm{NCH}\left(\mathrm{CH}_{3}\right)_{2}\right], 24.1\left(\mathrm{CH}_{2}\right), 26.4\left(\mathrm{CH}_{2}\right)$, $47.6\left[\mathrm{NCH}\left(\mathrm{CH}_{3}\right)_{2}\right], 58.3\left(\mathrm{NCH}_{2}\right)$. 
Synthesis of the formamidinium salts 1 . The trisubstituted hydrazine $(5.0 \mathrm{mmol})$ was dissolved in $10 \mathrm{~mL}$ of thf. The solution was cooled to $-78^{\circ} \mathrm{C}$, and $2.0 \mathrm{~mL}$ of a $2.5 \mathrm{M}$ solution of butyllithium in hexanes $(5.0 \mathrm{mmol})$ were added slowly. The solution was warmed to room temperature, stirred during half an hour, then cooled to $-78^{\circ} \mathrm{C}$. Chlorotrimethylsilane $(0.7 \mathrm{~mL}$, $5.5 \mathrm{mmol}$ ) was then added. The solution was warmed to room temperature, stirred for 30 minutes, and concentrated under vacuum. The residue was extracted with pentane (3x10 mL), and the filtrate was concentrated to afford the silylhydrazine, which was dissolved in dichloromethane $(10 \mathrm{~mL})$. The solution was cooled to $-50^{\circ} \mathrm{C}$, and added to a cold suspension $\left(-50^{\circ} \mathrm{C}\right)$ of $\mathrm{N}, \mathrm{N}$-diisopropylchloroformamidinium chloride $(0.70 \mathrm{~g}, 3.8 \mathrm{mmol})$ in dichloromethane $(10 \mathrm{~mL})$. The mixture was warmed slowly to room temperature, and stirred overnight. The resulting solution was concentrated, and the residue was washed with pentane (3x10 mL), and dried, to afford the formamidinium chloride $\mathbf{1 .} \mathbf{C l}^{-}$.

1a.Cl: yield 68\% ${ }^{1} \mathrm{H}$ NMR $\left(\mathrm{CD}_{3} \mathrm{CN}\right): \delta 1.26\left[\mathrm{~d},{ }^{3} J_{\mathrm{HH}}=6.4 \mathrm{~Hz}, 6 \mathrm{H}, \mathrm{NCH}\left(\mathrm{CH}_{3}\right)_{2}\right], 1.38[\mathrm{~d}$, $\left.{ }^{3} J_{\mathrm{HH}}=6.7 \mathrm{~Hz}, 6 \mathrm{H}, \mathrm{NCH}\left(\mathrm{CH}_{3}\right)_{2}\right], 1.44\left[\mathrm{~d},{ }^{3} J_{\mathrm{HH}}=7.3 \mathrm{~Hz}, 6 \mathrm{H}, \mathrm{NCH}\left(\mathrm{CH}_{3}\right)_{2}\right], 2.63[\mathrm{~s}, 6 \mathrm{H}$, $\mathrm{N}\left(\mathrm{CH}_{3}\right)_{2}$ ], 3.94 [sept, ${ }^{3} J_{\mathrm{HH}}=6.7 \mathrm{~Hz}, 1 \mathrm{H}, \mathrm{NCH}\left(\mathrm{CH}_{3}\right)_{2}$ ], 4.07 [sept, ${ }^{3} J_{\mathrm{HH}}=7.3 \mathrm{~Hz}, 1 \mathrm{H}$, $\mathrm{NCH}\left(\mathrm{CH}_{3}\right)_{2}$ ], 5.75 [sept, $\left.{ }^{3} J_{\mathrm{HH}}=6.4 \mathrm{~Hz}, 1 \mathrm{H}, \mathrm{NCH}\left(\mathrm{CH}_{3}\right)_{2}\right], 7.36\left(\mathrm{~s}, 1 \mathrm{H}, \mathrm{NCH}=\mathrm{N}^{+}\right) ;{ }^{13} \mathrm{C}\left\{{ }^{1} \mathrm{H}\right\}$ ( $\left.\mathrm{CD}_{3} \mathrm{CN}\right): 19.2\left[\mathrm{NCH}\left(\mathrm{CH}_{3}\right)_{2}\right], 22.8\left[\mathrm{NCH}\left(\mathrm{CH}_{3}\right)_{2}\right], 23.1\left[\mathrm{NCH}\left(\mathrm{CH}_{3}\right)_{2}\right], 42.5\left[\mathrm{~N}\left(\mathrm{CH}_{3}\right)_{2}\right], 49.1$ [NCH$\left.\left(\mathrm{CH}_{3}\right)_{2}\right], 50.2\left[\mathrm{NCH}\left(\mathrm{CH}_{3}\right)_{2}\right], 51.4\left[\mathrm{NCH}\left(\mathrm{CH}_{3}\right)_{2}\right], 149.0\left(\mathrm{NCH}=\mathrm{N}^{+}\right)$.

1b.Cl': yield 89\%. ${ }^{1} \mathrm{H}$ NMR $\left(\mathrm{CD}_{3} \mathrm{CN}\right): \delta 1.29$ [d, $\left.{ }^{3} \mathrm{~J}_{\mathrm{HH}}=6.4 \mathrm{~Hz}, 6 \mathrm{H}, \mathrm{NCH}\left(\mathrm{CH}_{3}\right)_{2}\right], 1.36[\mathrm{~d}$, $\left.{ }^{3} J_{\mathrm{HH}}=7.0 \mathrm{~Hz}, 6 \mathrm{H}, \mathrm{NCH}\left(\mathrm{CH}_{3}\right)_{2}\right], 1.41\left[\mathrm{~d},{ }^{3} J_{\mathrm{HH}}=7.0 \mathrm{~Hz}, 6 \mathrm{H}, \mathrm{NCH}\left(\mathrm{CH}_{3}\right)_{2}\right], 1.5-1.8(\mathrm{~m}, 6 \mathrm{H}$, $\mathrm{CH}_{2}$ ), 2.8-3.0 (m, $4 \mathrm{H}, \mathrm{NCH}_{2}$ ), $3.93\left[\mathrm{sept},{ }^{3} J_{\mathrm{HH}}=7.0 \mathrm{~Hz}, 1 \mathrm{H}, \mathrm{NCH}\left(\mathrm{CH}_{3}\right)_{2}\right.$ ], 4.11 [sept, ${ }^{3} J_{\mathrm{HH}}$ $\left.=7.0 \mathrm{~Hz}, 1 \mathrm{H}, \mathrm{NCH}\left(\mathrm{CH}_{3}\right)_{2}\right], 5.79\left[\mathrm{sept},{ }^{3} \mathrm{~J}_{\mathrm{HH}}=6.4 \mathrm{~Hz}, 1 \mathrm{H}, \mathrm{NCH}\left(\mathrm{CH}_{3}\right)_{2}\right], 7.26(\mathrm{~s}, 1 \mathrm{H}$, $\left.\mathrm{NCH}=\mathrm{N}^{+}\right) ;{ }^{13} \mathrm{C}\left\{{ }^{1} \mathrm{H}\right\}\left(\mathrm{CD}_{3} \mathrm{CN}\right): 20.5\left[\mathrm{NCH}\left(\mathrm{CH}_{3}\right)_{2}\right], 23.8\left(\mathrm{CH}_{2}\right), 24.3\left[\mathrm{NCH}\left(\mathrm{CH}_{3}\right)_{2}\right], 24.5$ [NCH$\left.\left(\mathrm{CH}_{3}\right)_{2}\right], 26.4\left(\mathrm{CH}_{2}\right), 51.0\left[\mathrm{NCH}\left(\mathrm{CH}_{3}\right)_{2}\right], 51.3\left[\mathrm{NCH}\left(\mathrm{CH}_{3}\right)_{2}\right], 52.8\left[\mathrm{NCH}\left(\mathrm{CH}_{3}\right)_{2}\right], 53.6$ $\left(\mathrm{NCH}_{2}\right), 149.4\left(\mathrm{NCH}=\mathrm{N}^{+}\right)$.

In order to increase the solubility of the formamidinium salts, the chloride was exchanged for tetraphenylborate as counter-anion. The formamidinium chloride $\mathbf{1 . C l ^ { - }}(2.7 \mathrm{mmol})$ was dissolved in acetonitrile $(20 \mathrm{~mL})$ and treated with sodium tetraphenylborate $(0.91 \mathrm{~g}, 2.7$ 
mmol) at room temperature. After stirring for 30 minutes, the solution was allowed to settle, and filtered. The filtrate was then concentrated to afford the formamidinium tetraphenylborate $\mathbf{1 . B P h}{ }_{4}{ }^{-}$as a white powder, which was crystallized from a dichloromethane/ether mixture at $-30^{\circ} \mathrm{C}$.

1a.BPh ${ }^{-}:$yield $81 \%$.

$\mathbf{1 b . B P h}_{\mathbf{4}}{ }^{-}$: Single crystals suitable for an X-ray diffraction study were grown from a saturated dichloromethane solution at $-30^{\circ} \mathrm{C}$. Yield $68 \%$; mp $212{ }^{\circ} \mathrm{C}$; ${ }^{1} \mathrm{H}$ NMR $\left(\mathrm{CD}_{3} \mathrm{CN}\right)$ : $\delta 1.34-1.40$ [m, $\left.18 \mathrm{H}, \mathrm{NCH}\left(\mathrm{CH}_{3}\right)_{2}\right], 1.5-1.8\left(\mathrm{~m}, 6 \mathrm{H}, \mathrm{CH}_{2}\right), 2.7-3.0\left(\mathrm{~m}, 4 \mathrm{H}, \mathrm{NCH}_{2}\right), 3.92$ [sept, ${ }^{3} J_{\mathrm{HH}}=$ $6.4 \mathrm{~Hz}, 1 \mathrm{H}, \mathrm{NCH}\left(\mathrm{CH}_{3}\right)_{2}$ ], 4.07 [sept, ${ }^{3} J_{\mathrm{HH}}=7.0 \mathrm{~Hz}, 1 \mathrm{H}, \mathrm{NCH}\left(\mathrm{CH}_{3}\right)_{2}$ ], 5.77 [sept, ${ }^{3} J_{\mathrm{HH}}=$ $7.0 \mathrm{~Hz}, 1 \mathrm{H}, \mathrm{NCH}\left(\mathrm{CH}_{3}\right)_{2}$ ], $6.87\left(\mathrm{~m}, 4 \mathrm{H}, \mathrm{BPh}_{4}\right), 7.02\left(\mathrm{~m}, 8 \mathrm{H}, \mathrm{BPh}_{4}\right), 7.12\left(\mathrm{~s}, 1 \mathrm{H}, \mathrm{NCH}=\mathrm{N}^{+}\right)$, $7.33\left(\mathrm{~m}, 8 \mathrm{H}, \mathrm{BPh}_{4}\right) ;{ }^{13} \mathrm{C}\left\{{ }^{1} \mathrm{H}\right\}\left(\mathrm{CD}_{3} \mathrm{CN}\right): 20.4$ [NCH$\left.\left(\mathrm{CH}_{3}\right)_{2}\right], 23.8\left(\mathrm{CH}_{2}\right), 24.3\left[\mathrm{NCH}\left(\mathrm{CH}_{3}\right)_{2}\right]$, $24.6\left[\mathrm{NCH}\left(\mathrm{CH}_{3}\right)_{2}\right], 26.4\left(\mathrm{CH}_{2}\right), 50.9\left[\mathrm{NCH}\left(\mathrm{CH}_{3}\right)_{2}\right], 51.2$ [ $\left.\mathrm{NCH}\left(\mathrm{CH}_{3}\right)_{2}\right], 52.9\left[\mathrm{NCH}\left(\mathrm{CH}_{3}\right)_{2}\right]$, $53.6\left(\mathrm{NCH}_{2}\right), 123.1\left(\mathrm{BPh}_{4}\right), 126.9\left(\mathrm{BPh}_{4}\right), 137.1\left(\mathrm{BPh}_{4}\right), 149.0\left(\mathrm{NCH}=\mathrm{N}^{+}\right), 166.1\left(\mathrm{q},{ }^{1} J_{\mathrm{BC}}=\right.$ $\left.50 \mathrm{~Hz}, \mathrm{BPh}_{4}\right)$.

Deprotonation of the formamidinium salts.

A solution of LiHMDS.OEt 2 (27mg, $0.12 \mathrm{mmol})$ in deuterated thf $(0.4 \mathrm{~mL})$ is added at $-78^{\circ} \mathrm{C}$ on a solution of formamidinium tetraphenylborate $(0.10 \mathrm{mmol})$ in deuterated thf $(0.3 \mathrm{~mL})$ placed in an NMR tube kept at $-78^{\circ} \mathrm{C}$. After mixing, the reaction mixture is directly analyzed by NMR spectroscopy at low temperature.

2a: ${ }^{1} \mathrm{H}$ NMR (thf-D $8,240 \mathrm{~K}$ ): $\delta 1.07\left[\mathrm{~d},{ }^{3} J_{\mathrm{HH}}=6.8 \mathrm{~Hz}, 6 \mathrm{H}, \mathrm{NCH}\left(\mathrm{CH}_{3}\right)_{2}\right], 1.17\left[\mathrm{~d},{ }^{3} J_{\mathrm{HH}}=6.7\right.$ $\left.\mathrm{Hz}, 6 \mathrm{H}, \mathrm{NCH}\left(\mathrm{CH}_{3}\right)_{2}\right], 1.24\left[\mathrm{~d},{ }^{3} J_{\mathrm{HH}}=6.2 \mathrm{~Hz}, 6 \mathrm{H}, \mathrm{NCH}\left(\mathrm{CH}_{3}\right)_{2}\right], 2.43\left[\mathrm{~s}, 6 \mathrm{H}, \mathrm{N}\left(\mathrm{CH}_{3}\right)_{2}\right]$, 3.52 [sept, ${ }^{3} J_{\mathrm{HH}}=6.8 \mathrm{~Hz}, 1 \mathrm{H}, \mathrm{NCH}\left(\mathrm{CH}_{3}\right)_{2}$ ], 3.65 [sept, ${ }^{3} J_{\mathrm{HH}}=6.2 \mathrm{~Hz}, 1 \mathrm{H}, \mathrm{NCH}\left(\mathrm{CH}_{3}\right)_{2}$ ], 5.96 [sept, $\left.{ }^{3} J_{\mathrm{HH}}=6.7 \mathrm{~Hz}, 1 \mathrm{H}, \mathrm{NCH}\left(\mathrm{CH}_{3}\right)_{2}\right] ;{ }^{13} \mathrm{C}\left\{{ }^{1} \mathrm{H}\right\}$ (thf-D $\left.8,240 \mathrm{~K}\right): 22.2\left[\mathrm{NCH}\left(\mathrm{CH}_{3}\right)_{2}\right]$, $26.3\left[\mathrm{NCH}\left(\mathrm{CH}_{3}\right)_{2}\right], \quad 27.0 \quad\left[\mathrm{NCH}\left(\mathrm{CH}_{3}\right)_{2}\right], \quad 44.1 \quad\left[\mathrm{~N}\left(\mathrm{CH}_{3}\right)_{2}\right], \quad 46.1 \quad\left[\mathrm{NCH}\left(\mathrm{CH}_{3}\right)_{2}\right], \quad 47.6$ [NCH($\left.\left(\mathrm{CH}_{3}\right)_{2}\right], 49.6\left[\mathrm{NCH}\left(\mathrm{CH}_{3}\right)_{2}\right], 228.9(\mathrm{NCN})$. 
2b: ${ }^{1} \mathrm{H}$ NMR (thf-D, $\left.258 \mathrm{~K}\right): \delta 1.10\left[\mathrm{~d},{ }^{3} J_{\mathrm{HH}}=6.1 \mathrm{~Hz}, 6 \mathrm{H}, \mathrm{NCH}\left(\mathrm{CH}_{3}\right)_{2}\right], 1.16\left[\mathrm{~d},{ }^{3} J_{\mathrm{HH}}=6.1\right.$ $\mathrm{Hz}, 6 \mathrm{H}, \mathrm{NCH}\left(\mathrm{CH}_{3}\right)_{2}$ ], 1.23 [d, $\left.{ }^{3} J_{\mathrm{HH}}=6.1 \mathrm{~Hz}, 6 \mathrm{H}, \mathrm{NCH}\left(\mathrm{CH}_{3}\right)_{2}\right], 1.4-1.6$ (m, 6H, $\left.\mathrm{CH}_{2}\right)$, 2.6$2.8\left(\mathrm{~m}, 4 \mathrm{H}, \mathrm{NCH}_{2}\right), 3.50$ [sept, ${ }^{3} J_{\mathrm{HH}}=6.1 \mathrm{~Hz}, 1 \mathrm{H}, \mathrm{NCH}\left(\mathrm{CH}_{3}\right)_{2}$ ], 3.67 [sept, ${ }^{3} J_{\mathrm{HH}}=6.1 \mathrm{~Hz}$, $1 \mathrm{H}, \mathrm{NCH}\left(\mathrm{CH}_{3}\right)_{2}$ ], 6.05 [sept, $\left.{ }^{3} J_{\mathrm{HH}}=6.1 \mathrm{~Hz}, 1 \mathrm{H}, \mathrm{NCH}\left(\mathrm{CH}_{3}\right)_{2}\right] ;{ }^{13} \mathrm{C}\left\{{ }^{1} \mathrm{H}\right\}$ (thf- $\left.\mathrm{D}_{8}, 258 \mathrm{~K}\right)$ : 21.0, 23.8, 25.5, 26.0, 46.1, 46.5, 48.5, 53.0, 228.0 (NCN). 1C was not observed.

Determination of the half-life times.

The half-life times of carbenes $\mathbf{2 a}$ and $\mathbf{2} \mathbf{b}$ were determined by ${ }^{1} \mathrm{H}$ NMR spectroscopy on the basis of the integrals of the peaks at $\delta 2.43$ and 6.05 respectively, using the solvent peak ( $\delta$ 1.73) as an internal standard.

3: ${ }^{1} \mathrm{H}$ NMR $\left(\mathrm{C}_{6} \mathrm{D}_{6}, 298 \mathrm{~K}\right): \delta 1.01\left[\mathrm{~d},{ }^{3} J_{\mathrm{HH}}=6.4 \mathrm{~Hz}, 12 \mathrm{H}, \mathrm{NCH}\left(\mathrm{CH}_{3}\right)_{2}\right], 1.28\left[\mathrm{~d},{ }^{3} J_{\mathrm{HH}}=6.4\right.$ $\mathrm{Hz}, 6 \mathrm{H}, \mathrm{NCH}\left(\mathrm{CH}_{3}\right)_{2}$ ], 3.28 [sept, ${ }^{3} J_{\mathrm{HH}}=6.4 \mathrm{~Hz}, 1 \mathrm{H}, \mathrm{NCH}\left(\mathrm{CH}_{3}\right)_{2}$ ], $3.79[\mathrm{br}, 2 \mathrm{H}$, $\left.\mathrm{NCH}\left(\mathrm{CH}_{3}\right)_{2}\right], 7.44$ (s, $\left.1 \mathrm{H}, \mathrm{NCHN}\right) ;{ }^{13} \mathrm{C}\left\{{ }^{1} \mathrm{H}\right\}\left(\mathrm{C}_{6} \mathrm{D}_{6}, 298 \mathrm{~K}\right): 22.2\left[\mathrm{NCH}\left(\mathrm{CH}_{3}\right)_{2}\right], 26.8$ [NCH( $\left.\left(\mathrm{CH}_{3}\right)_{2}\right], 45.5\left[\mathrm{NCH}\left(\mathrm{CH}_{3}\right)_{2}\right], 57.9$ [NCH$\left.\left(\mathrm{CH}_{3}\right)_{2}\right], 148.4(\mathrm{NCHN})$. Mass (EI): 170.

4: ${ }^{1} \mathrm{H}$ NMR (thf-D, $298 \mathrm{~K}$ ): $\delta 3.25$ (s, $3 \mathrm{H}, \mathrm{NCH}_{3}$ ), 7.07 (d, ${ }^{2} J_{\mathrm{HH}}=16 \mathrm{~Hz}, 1 \mathrm{H}, \mathrm{NCH}_{2}$ ), 7.39 $\left(\mathrm{d},{ }^{2} J_{\mathrm{HH}}=16 \mathrm{~Hz}, 1 \mathrm{H}, \mathrm{NCH}_{2}\right) ;{ }^{13} \mathrm{C}\left\{{ }^{1} \mathrm{H}\right\}($ thf-D, $298 \mathrm{~K}): 50.1\left(\mathrm{NCH}_{3}\right), 154.7\left(\mathrm{~N}=\mathrm{CH}_{2}\right)$.

5: To a suspension of formamidinium chloride $(19 \mathrm{mmol})$ in $40 \mathrm{~mL}$ of thf at $-78^{\circ} \mathrm{C}$ was added a solution of $\mathrm{LiHMDS} . O E t_{2}(25 \mathrm{mmol})$ in $20 \mathrm{~mL}$ of thf at $-78^{\circ} \mathrm{C}$. The mixture was slowly warmed to room temperature, then stirred for one hour. After evaporation of the solvents, pentane was added. The mixture was filtered over a pad of celite, and the filtrate was concentrated. The oxamidine was obtained after chromatography on silica gel (ether). Single crystals suitable for an X-ray diffraction study were grown from a saturated pentane solution at $-30^{\circ} \mathrm{C}$. Yield: $15 \%$; mp: $122^{\circ} \mathrm{C}$; Mass (EI): 338; ${ }^{1} \mathrm{H}$ NMR $\left(\mathrm{CDCl}_{3}, 298 \mathrm{~K}\right): \delta 1.02\left[\mathrm{~d},{ }^{3} J_{\mathrm{HH}}=\right.$ $\left.5.8 \mathrm{~Hz}, 6 \mathrm{H}, \mathrm{NCH}\left(\mathrm{CH}_{3}\right)_{2}\right], 1.03\left[\mathrm{~d},{ }^{3} J_{\mathrm{HH}}=5.8 \mathrm{~Hz}, 6 \mathrm{H}, \mathrm{NCH}\left(\mathrm{CH}_{3}\right)_{2}\right], 1.10\left[\mathrm{~d},{ }^{3} J_{\mathrm{HH}}=6.4 \mathrm{~Hz}\right.$, $\left.6 \mathrm{H}, \mathrm{NCH}\left(\mathrm{CH}_{3}\right)_{2}\right], 1.12$ [d, $\left.{ }^{3} J_{\mathrm{HH}}=6.4 \mathrm{~Hz}, 6 \mathrm{H}, \mathrm{NCH}\left(\mathrm{CH}_{3}\right)_{2}\right], 1.44\left[\mathrm{~d},{ }^{3} J_{\mathrm{HH}}=6.7 \mathrm{~Hz}, 6 \mathrm{H}\right.$, $\mathrm{NCH}\left(\mathrm{CH}_{3}\right)_{2}$ ], 1.51 [d, ${ }^{3} J_{\mathrm{HH}}=6.7 \mathrm{~Hz}, 6 \mathrm{H}, \mathrm{NCH}\left(\mathrm{CH}_{3}\right)_{2}$ ], 3.2 [br, $2 \mathrm{H}, \mathrm{NCH}\left(\mathrm{CH}_{3}\right)_{2}$ ], 3.7 [br, 4 $\left.\mathrm{H}, \mathrm{NCH}\left(\mathrm{CH}_{3}\right)_{2}\right] ;{ }^{13} \mathrm{C}\left\{{ }^{1} \mathrm{H}\right\}\left(\mathrm{CDCl}_{3}, 298 \mathrm{~K}\right): 20.1\left[\mathrm{NCH}\left(\mathrm{CH}_{3}\right)_{2}\right], 21.1\left[\mathrm{NCH}\left(\mathrm{CH}_{3}\right)_{2}\right], 21.7$ $\left[\mathrm{NCH}\left(\mathrm{CH}_{3}\right)_{2}\right], \quad 22.1 \quad\left[\mathrm{NCH}\left(\mathrm{CH}_{3}\right)_{2}\right], \quad 25.7 \quad\left[\mathrm{NCH}\left(\mathrm{CH}_{3}\right)_{2}\right], \quad 26.4 \quad\left[\mathrm{NCH}\left(\mathrm{CH}_{3}\right)_{2}\right], \quad 45.5$ 
[NCH$\left.\left(\mathrm{CH}_{3}\right)_{2}\right], 47.5\left[\mathrm{NCH}\left(\mathrm{CH}_{3}\right)_{2}\right], 49.0\left[\mathrm{NCH}\left(\mathrm{CH}_{3}\right)_{2}\right], 148.2$ (NCN). Anal. calcd for $\mathrm{C}_{20} \mathrm{H}_{42} \mathrm{~N}_{4}$ : C 70.95, H 12.50, N 16.55; found: C 69.15, H 12.42, N 15.76.

Determination of the 3/5 ratio in the mixtures resulting from the fragmentation of carbenes $2 \boldsymbol{a}$ and $2 \boldsymbol{b}$.

The molar ratio of $\mathbf{3} / \mathbf{5}$ resulting from $2 \mathbf{a}$ (96/4) was measured by combined ${ }^{1} \mathrm{H}$ NMR and GC analysis of the corresponding reaction mixture. The molar ratio of $\mathbf{3} / \mathbf{5}$ resulting from $\mathbf{2 b}(6 / 4)$ could then be determined by GC analysis $\left({ }^{1} \mathrm{H}\right.$ NMR analysis was prevented in this case by the overlap of different signals).

\section{Computational details and calculation results}

Calculations were performed with the Gaussian 98 program $^{\mathrm{S3}, \mathrm{S} 4}$ using the Density Functional Theory method. ${ }^{\text {S5 }}$ The various structures were fully optimized at B3LYP level. ${ }^{\text {S6 }}$ This functional is built with Becke's three parameter exchange functional ${ }^{\text {S6a }}$ and the LeeYang-Parr correlation functional. ${ }^{\mathrm{S} 6 \mathrm{c}}$ The 6-31G(d,p) basis set was used. All atoms were augmented with a single set of polarization functions. The second derivatives were calculated in order to determine if a minimum or a transition state (one negative eigenvalue) existed for the resulting geometry. The connection between the transition states $\mathbf{T} \mathbf{S}_{\text {frag, }}, \mathbf{T S M} \mathbf{M}_{\text {frag }}$ and the corresponding minima was confirmed by IRC calculations. All total energies have been zeropoint energy (ZPE) and temperature corrected using unscaled density functional frequencies.

The following notations have been used throughout the manuscript:

$E_{\text {tot }}(\mathrm{ZPE})$ : sum of electronic and zero point energies

$\mathrm{H}$ (ZPE): sum of electronic and thermal enthalpies

$\mathrm{G}$ (ZPE): sum of electronic and thermal free energies calculated at $25^{\circ} \mathrm{C}$

S: total entropy 


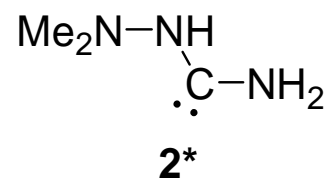

For the singlet carbene $\mathrm{Me}_{2} \mathrm{~N}(\mathrm{NH})-\mathrm{C}-\mathrm{NH}_{2} \mathbf{2}^{*}$, four minima were found on the potential energy surface. The different conformers are drawn in Figure S1 and the geometrical parameters are presented in Table S1.
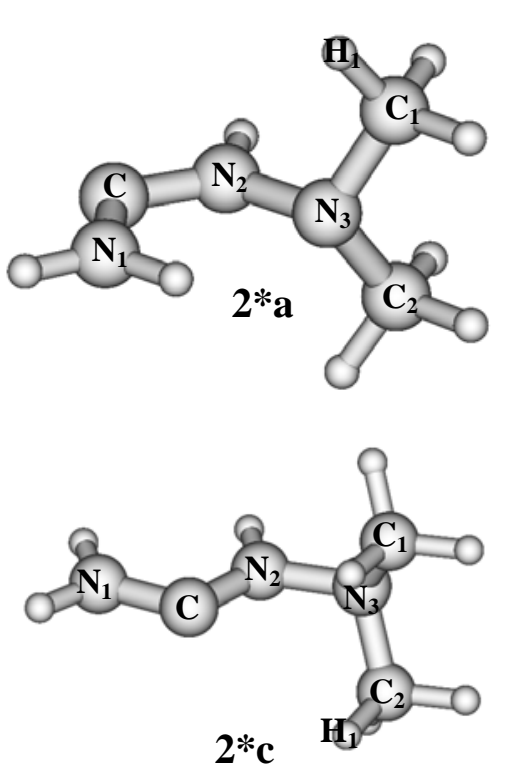
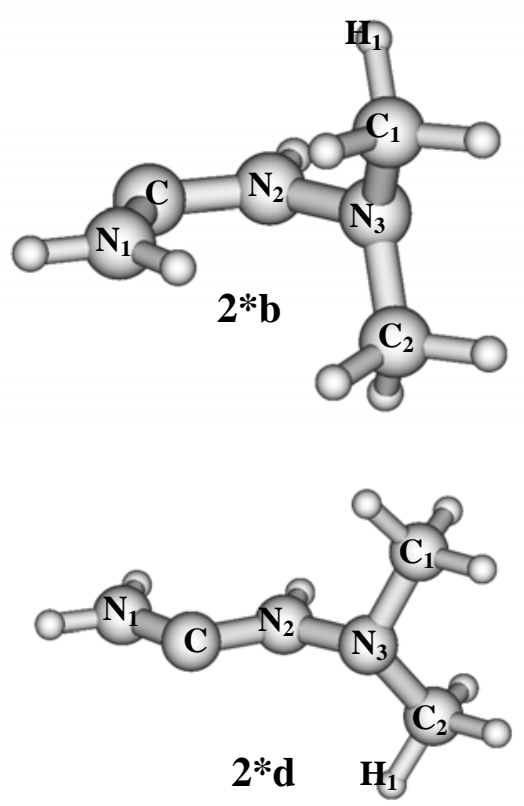

Figure S1: Different minima found on the potential energy surface for the singlet carbene $\mathrm{Me}_{2} \mathrm{~N}(\mathrm{NH})-\mathrm{C}-\mathrm{NH}_{2}(2 *)$.

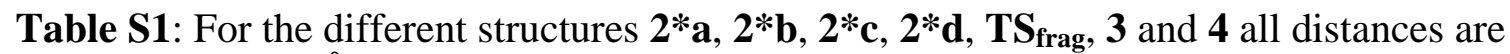
given in angstroms $(\AA)$, the bond angles in degrees $\left(^{\circ}\right)$ and the energy differences in $\mathrm{kcal} / \mathrm{mol}$.

\begin{tabular}{|c|c|c|c|c|c|c|c|}
\hline & $2 * a$ & $2 * b$ & $2 * \mathrm{c}$ & $2 * d$ & $\mathbf{T S}_{\text {frag }}$ & 3 & 4 \\
\hline $\mathrm{N}_{1} \mathrm{C}$ & 1.341 & 1.345 & 1.342 & 1.345 & 1.341 & 1.382 & $\mathbf{N}_{3} \mathbf{C}_{2}: 1.452$ \\
\hline $\mathrm{N}_{2} \mathrm{C}$ & 1.347 & 1.343 & 1.334 & 1.337 & 1.243 & 1.276 & $\mathbf{N}_{3} \mathbf{C}_{2}: 1.267$ \\
\hline $\mathbf{N}_{2} \mathbf{N}_{3}$ & 1.431 & 1.436 & 1.422 & 1.407 & 1.976 & 1 & I \\
\hline $\mathbf{C}_{1} \mathbf{H}_{1}$ & 3.354 & 3.263 & 2.762 & 3.338 & 1.251 & 1.094 & I \\
\hline $\mathrm{N}_{1} \mathrm{CN}_{2}$ & 112.48 & 115.17 & 112.76 & 111.64 & 132.77 & 129.41 & 117.71 \\
\hline $\mathrm{CN}_{2} \mathbf{N}_{3}$ & 125.90 & 132.59 & 122.99 & 121.65 & 135.62 & I & I \\
\hline$\Sigma \mathbf{N}_{1}$ & 360.0 & 360.0 & 360.0 & 360.0 & 360.0 & 350.0 & I \\
\hline$\Sigma \mathbf{N}_{2}$ & 360.0 & 360.0 & 360.0 & 360.0 & I & I & I \\
\hline$\Sigma \mathbf{N}_{3}$ & 332.7 & 343.7 & 333.3 & 333.5 & l & I & I \\
\hline$\Delta \mathbf{E}$ & -2.6 & 3.9 & 0 & 1.6 & 26.0 & \multicolumn{2}{|c|}{$3+4:-30.6^{a}$} \\
\hline
\end{tabular}

${ }^{a}$ calculated from the energies of the compounds 3 and $\mathbf{4}$ separately optimized. $-33.6 \mathrm{kcal} / \mathrm{mol}$ when the compounds $\mathbf{3}$ and $\mathbf{4}$ are carried out in the same calculation (correction of the basis set effect). 
For the $\beta$-fragmentation process of $\mathbf{2}^{*}$, a cyclic transition state $\mathbf{T S}_{\text {frag }}$ has been located on the potential energy surface. The latter $(2 * \mathbf{c})$ as well as the connected minima (3 and 4) are drawn in Figure S2. The geometrical parameters of $2 * \mathbf{c}, \mathbf{T S}_{\text {frag }}, \mathbf{3}$ and $\mathbf{4}$ are presented in Table S1. Note that the reactive conformer $2 * \mathbf{c}$ is $2.6 \mathrm{kcal} / \mathrm{mol}$ higher in energy than the most stable conformer $2 *$ a but the barrier to rotation about $\mathrm{C}_{\text {carbene }} \mathrm{N}$ bonds responsible for the interconversion between $2 * \mathbf{a}$ and $2 * \mathrm{c}$ can be predicted to be of about 10 $\mathrm{kcal} / \mathrm{mol} .{ }^{\mathrm{S} 7}$ For the $\beta$-fragmentation process of $2 *$ the entropic effects are weak. No difference is observed between $\Delta \mathrm{E}^{\#}$ and $\Delta \mathrm{G}^{\#}$ for the $\beta$-fragmentation reaction.

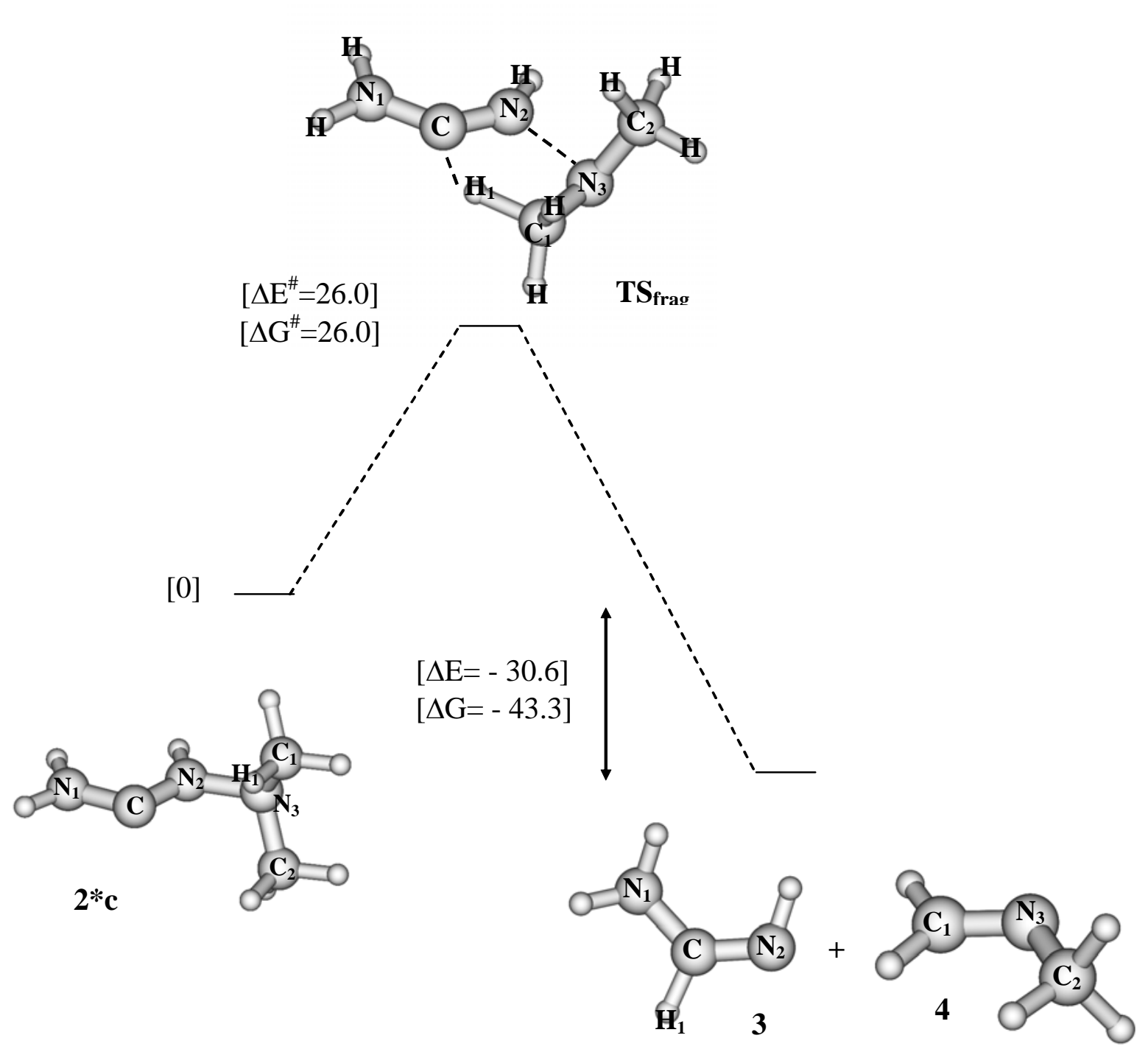

Figure S2: Transition state of the $\beta$-fragmentation reaction and minima connected to the latter. Energy differences in $\mathrm{kcal} / \mathrm{mol}$. 
For the radical 6*, 2 minima were found on the potential energy surface. The 2 structures are drawn in Figure S3. The geometrical parameters and the energy differences are presented in Table S2.
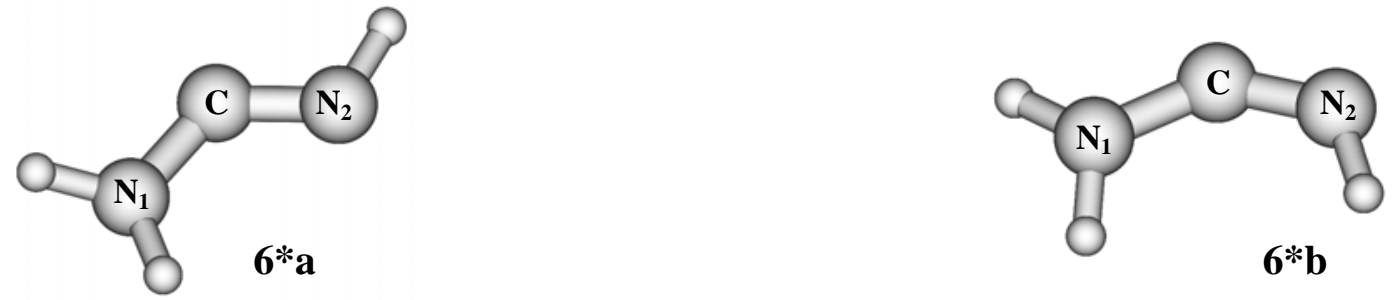

Figure S3: trans and cis isomers for the radical 6*.

Table S2: For the two structures $\mathbf{6 * a}, \mathbf{6} * \mathbf{b}$ all distances are given in angstroms $(\AA)$, the bond angles in degrees $\left(^{\circ}\right)$ and the energy differences in $\mathrm{kcal} / \mathrm{mol}$.

\begin{tabular}{ccc}
\hline & 6*a & 6*b \\
\hline $\mathbf{N}_{\mathbf{1}} \mathbf{C}$ & 1.350 & 1.354 \\
$\mathbf{N}_{\mathbf{2}} \mathbf{C}$ & 1.255 & 1.237 \\
$\mathbf{N}_{\mathbf{1}} \mathbf{C N}_{\mathbf{2}}$ & 128.76 & 138.65 \\
$\boldsymbol{\Sigma N}_{\mathbf{1}}$ & 354.6 & 353.6 \\
$\Delta \mathbf{E}$ & 0 & 6.1 \\
\hline
\end{tabular}

The fragmentation of the singlet carbene $2^{*}$ can lead to the two radicals $\mathbf{M e}_{2} \mathbf{N}$ and $\mathbf{6}^{*}$. The $\mathrm{N}-\mathrm{N}$ bond energy of $2^{*}$ has been estimated to be the energy required for homolytic fragmentation of $2 *$. Accordingly, the N-N energy of $2 *$ a was estimated at $27.8 \mathrm{kcal} / \mathrm{mol}$ $\left(E_{6 * a}+E_{M e 2 N}-E_{2 * a}\right)$ or $14.4 \mathrm{kcal} / \mathrm{mol}$ (free energy: $\left.G_{6 * a}+G_{M e 2 N}-G_{2 * a}\right)$ and the N-N energy of $2 * \mathbf{c}$ was estimated at $31.3 \mathrm{kcal} / \mathrm{mol}\left(\mathrm{E}_{\mathbf{6} * \mathbf{b}}+\mathrm{E}_{\mathbf{M e} 2 \mathrm{~N}}-\mathrm{E}_{2 * \mathbf{c}}\right)$ or $17.8 \mathrm{kcal} / \mathrm{mol}$ (free energy: $\mathrm{G}_{6 * \mathbf{b}}+\mathrm{G}_{\mathrm{Me2N}}-\mathrm{G}_{2{ }^{*} \mathrm{c}}$ ). The decrease of the N-N energy of $2 *$, when taking into account the free energy, shows that the entropic factors are in favor of the dissociative radical pathway. 
Cartesian coordinates for all the compounds, Zero point Energy, Enthalpy, free energy in ua and entropy in $\mathrm{cal} / \mathrm{mol} / \mathrm{K}$ :

2*a:

$\begin{array}{lrrr}\mathrm{N} & -0.356617 & -0.004230 & 0.813727 \\ \mathrm{C} & -1.692931 & -0.003161 & 0.644734 \\ \mathrm{~N} & -2.046097 & 0.001799 & -0.648626 \\ \mathrm{~N} & 0.621337 & 0.000400 & -0.231167 \\ \mathrm{H} & 0.007334 & -\Theta .005723 & 1.761212 \\ \mathrm{H} & -3.024461 & 0.003075 & -0.880631 \\ \mathrm{H} & -1.370253 & 0.003720 & -1.410465 \\ \mathrm{C} & 1.428454 & 1.216412 & -0.159798 \\ \mathrm{C} & 1.432688 & -1.213064 & -0.165393 \\ \mathrm{H} & 2.139803 & -1.222422 & -1.000936 \\ \mathrm{H} & 2.010788 & -1.292083 & 0.773716 \\ \mathrm{H} & 0.781108 & -2.085677 & -0.245863 \\ \mathrm{H} & 2.135903 & 1.231751 & -0.994981 \\ \mathrm{H} & 0.774037 & 2.087179 & -0.236627 \\ \mathrm{H} & 2.006113 & 1.293270 & 0.779774\end{array}$

$E_{\text {tot }}(\mathrm{ZPE}):-283.786737$ ua (B3LYP)

$E_{\text {tot }}$ (ZPE): -283.786741 иа (UB3LYP)

H (ZPE): -283.778386 иа

G (ZPE): -283.817752 иа

S: $82.85 \mathrm{cal} / \mathrm{mol} / \mathrm{K}$

2*b:

$\begin{array}{lrrr}\mathrm{N} & 0.394612 & -0.003464 & 1.023460 \\ \mathrm{C} & 1.645535 & -0.001887 & 0.534015 \\ \mathrm{~N} & 1.734616 & 0.001945 & -0.808053 \\ \mathrm{~N} & -0.895693 & -0.000610 & 0.393117 \\ \mathrm{H} & 0.308224 & -0.005675 & 2.028053 \\ \mathrm{H} & 2.655946 & 0.002254 & -1.213214 \\ \mathrm{H} & 0.964232 & 0.002788 & -1.470383 \\ \mathrm{C} & -1.211131 & 1.231612 & -0.316608 \\ \mathrm{C} & -1.213284 & -1.228981 & -0.322287 \\ \mathrm{H} & -2.291492 & -1.251422 & -0.517425 \\ \mathrm{H} & -0.696751 & -1.356332 & -1.289977 \\ \mathrm{H} & -0.960723 & -2.083772 & 0.308992 \\ \mathrm{H} & -2.289443 & 1.257219 & -0.510820 \\ \mathrm{H} & -0.956466 & 2.083017 & 0.318398 \\ \mathrm{H} & -0.694996 & 1.362371 & -1.284005\end{array}$

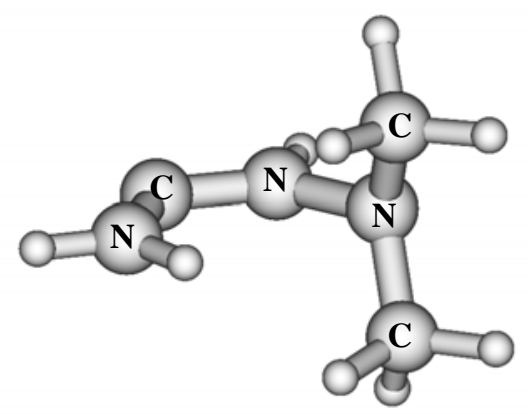

$E_{\text {tot }}(\mathrm{ZPE}):-283.776407$ ua

H (ZPE): -283.767858 иа

G (ZPE): -283.807276 иа

S: $82.96 \mathrm{cal} / \mathrm{mol} / \mathrm{K}$ 
2*c:

$\begin{array}{rrrr}\mathrm{N} & 0.400939 & -0.000904 & 0.572810 \\ \mathrm{C} & 1.215164 & 0.001424 & -0.483580 \\ \mathrm{~N} & 2.512728 & -0.000104 & -0.139196 \\ \mathrm{~N} & -1.016380 & -0.000239 & 0.457880 \\ \mathrm{H} & 0.703245 & -0.003083 & 1.550662 \\ \mathrm{C} & -1.477040 & 1.215827 & -0.215443 \\ \mathrm{C} & -1.477688 & -1.215352 & -0.216934 \\ \mathrm{H} & -2.571951 & -1.211914 & -0.230403 \\ \mathrm{H} & -1.100603 & -1.296645 & -1.246977 \\ \mathrm{H} & -1.142140 & -2.088701 & 0.349061 \\ \mathrm{H} & -2.571316 & 1.213765 & -0.227241 \\ \mathrm{H} & -1.139490 & 2.088218 & 0.350811 \\ \mathrm{H} & -1.101599 & 1.297794 & -1.246061 \\ \mathrm{H} & 3.210722 & 0.001187 & -0.863761 \\ \mathrm{H} & 2.869511 & -0.003279 & 0.819199\end{array}$

$E_{\text {tot }}(\mathrm{ZPE}):-283.782588$ ua

$E_{\text {tot }}$ (ZPE): -283.782588 ua (UB3LYP)

H (ZPE): -283.774125 иа

G (ZPE): -283.813399 иа

S: $82.66 \mathrm{cal} / \mathrm{mol} / \mathrm{K}$

$2 * d:$

$\begin{array}{lrrr}\mathrm{N} & 0.394799 & -0.039682 & 0.198316 \\ \mathrm{C} & 1.476002 & 0.018374 & -0.586492 \\ \mathrm{~N} & 2.612518 & -0.009225 & 0.132153 \\ \mathrm{~N} & -0.907434 & 0.001920 & -0.334261 \\ \mathrm{H} & 0.435860 & -0.075703 & 1.231975 \\ \mathrm{C} & -1.644254 & -1.198108 & 0.042314 \\ \mathrm{C} & -1.580838 & 1.232066 & 0.066077 \\ \mathrm{H} & -2.572597 & 1.263037 & -0.394471 \\ \mathrm{H} & -1.708035 & 1.324462 & 1.162331 \\ \mathrm{H} & -1.001261 & 2.085880 & -0.291495 \\ \mathrm{H} & -2.636770 & -1.166693 & -0.416267 \\ \mathrm{H} & -1.112039 & -2.075366 & -0.331833 \\ \mathrm{H} & -1.776426 & -1.304512 & 1.136842 \\ \mathrm{H} & 3.492151 & 0.027260 & -0.354335 \\ \mathrm{H} & 2.674476 & -0.063443 & 1.152396\end{array}$

$E_{\text {tot }}(\mathrm{ZPE}):-283.779980$ ua

H (ZPE): -283.778386 иа

G (ZPE): -283.811207 иа

S: $82.56 \mathrm{cal} / \mathrm{mol} / \mathrm{K}$

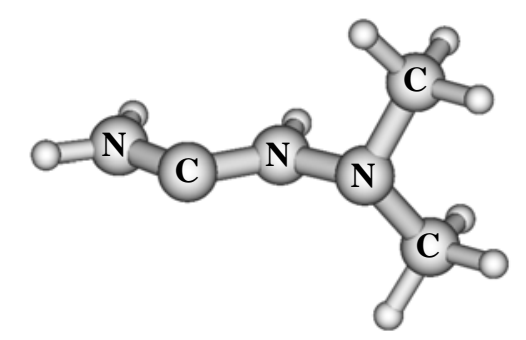


TS $_{\text {frag: }}$

$\begin{array}{lrrr}\mathrm{N} & 0.471751 & -\Theta .752216 & -0.363370 \\ \mathrm{C} & 1.305235 & 0.084694 & 0.025007 \\ \mathrm{~N} & 2.630340 & 0.026967 & 0.220498 \\ \mathrm{~N} & -1.307505 & 0.093840 & -0.516402 \\ \mathrm{H} & 0.512070 & -1.671450 & -0.779722 \\ \mathrm{C} & -2.028274 & -\Theta .759149 & 0.414702 \\ \mathrm{C} & -1.003880 & 1.344182 & -0.025680 \\ \mathrm{H} & -1.076031 & 2.142110 & -0.770941 \\ \mathrm{H} & -1.435011 & 1.623822 & 0.945289 \\ \mathrm{H} & 0.222913 & 1.242020 & 0.199237 \\ \mathrm{H} & -3.099044 & -0.524239 & 0.340393 \\ \mathrm{H} & -1.905712 & -1.812053 & 0.137439 \\ \mathrm{H} & -1.728939 & -\Theta .637070 & 1.469065 \\ \mathrm{H} & 3.127241 & 0.846575 & 0.527899 \\ \mathrm{H} & 3.181929 & -0.808216 & 0.062098\end{array}$

frequency: $\quad-720.059 \mathrm{~cm}^{-1}$

$E_{\text {tot }}(\mathrm{ZPE}):-283.741182$ ua

H (ZPE): -283.732788 ua, G (ZPE): -283.771907 ua, S: $82.33 \mathrm{cal} / \mathrm{mol} / \mathrm{K}$

3:

$\begin{array}{rrrr}\mathrm{N} & 1.172714 & -0.333765 & 0.017224 \\ \mathrm{C} & 0.123449 & 0.394412 & -0.002669 \\ \mathrm{~N} & -1.139510 & -0.147390 & -0.071799 \\ \mathrm{H} & 2.005507 & 0.253879 & -0.002318 \\ \mathrm{H} & 0.109579 & 1.493009 & -0.012770 \\ \mathrm{H} & -1.902776 & 0.396132 & 0.301576 \\ \mathrm{H} & -1.185437 & -1.141405 & 0.111554\end{array}$

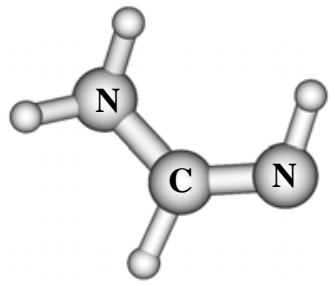

$E_{\text {tot }}$ (ZPE): -149.952567 ua

H (ZPE): -149.947950 иа, G (ZPE): -149.976982 ua, S: $61.10 \mathrm{cal} / \mathrm{mol} / \mathrm{K}$

4:

$\begin{array}{rrrr}\mathrm{N} & 0.139861 & -0.537770 & -0.000005 \\ \mathrm{C} & -1.147297 & 0.135371 & -0.000014 \\ \mathrm{C} & 1.181607 & 0.183044 & 0.000011 \\ \mathrm{H} & 1.167248 & 1.284509 & -0.000009 \\ \mathrm{H} & 2.162739 & -0.295404 & -0.000047 \\ \mathrm{H} & -1.081561 & 1.235637 & -0.000547 \\ \mathrm{H} & -1.716924 & -0.185860 & -0.879102 \\ \mathrm{H} & -1.716387 & -0.184979 & 0.879756\end{array}$

$E_{\text {tot }}(\mathrm{ZPE}):-133.880766$ ua

H (ZPE): -133.875963 иа, G (ZPE): -133.905494 иа, S: $62.15 \mathrm{cal} / \mathrm{mol} / \mathrm{K}$ 
$3+4$ in the same calculation at $\sim 3.7 \AA$ :

$E_{\text {tot }}$ (ZPE): -283.836227 ua (correction of the basis set effect)

$H$ (ZPE):-283.826009 ua (correction of the basis set effect)

$G(Z P E):-283.871837$ ua (correction of the basis set effect)

S: $96.45 \mathrm{cal} / \mathrm{mol} / \mathrm{K}$

\section{3 and 4 in separate calculations:}

$E_{\text {tot }}(\mathrm{ZPE}):-283.833333$ ua $\left[=E_{\text {tot }}^{3}(\mathrm{ZPE})+E_{\text {tot }}^{4}(\mathrm{ZPE})\right]$

$H(Z P E):-283.823913$ иа $\left[=H^{3}(Z P E)+H^{4}(Z P E)\right]$

$G(\mathrm{ZPE}):-283.882476$ иа $\left[=G^{3}(\mathrm{ZPE})+G^{4}(\mathrm{ZPE})\right]$

$S: 123.25 \mathrm{cal} / \mathrm{mol} / \mathrm{K}\left[=S^{3}+S^{4}\right]$

\section{$\mathrm{Me}_{2} \mathrm{~N}$ :}

$\begin{array}{lrrr}\mathrm{N} & 0.000045 & -0.638653 & -0.000092 \\ \mathrm{C} & 1.192821 & 0.171419 & -0.000081 \\ \mathrm{H} & 2.084594 & -0.461215 & -0.002309 \\ \mathrm{H} & 1.232244 & 0.836474 & -0.880449 \\ \mathrm{H} & 1.233772 & 0.831624 & 0.883841 \\ \mathrm{H} & -1.233473 & 0.833075 & -0.882776 \\ \mathrm{H} & -2.084424 & -0.461445 & 0.000865 \\ \mathrm{H} & -1.232738 & 0.834487 & 0.881795\end{array}$

$E_{\text {tot }}(\mathrm{ZPE}):-134.440279$ ua

H (ZPE): -134.434510 иа

G (ZPE): -134.467421 иа

S: $69.27 \mathrm{cal} / \mathrm{mol} / \mathrm{K}$

6*a:

$\begin{array}{rrrr}\mathrm{N} & -1.202691 & 0.231645 & 0.012368 \\ \mathrm{C} & -0.119138 & -0.401483 & 0.000897 \\ \mathrm{~N} & 1.141347 & 0.080672 & -0.049638 \\ \mathrm{H} & -2.060755 & -0.311882 & -0.018142 \\ \mathrm{H} & 1.304139 & 1.072827 & 0.094207 \\ \mathrm{H} & 1.900857 & -0.538264 & 0.179444\end{array}$

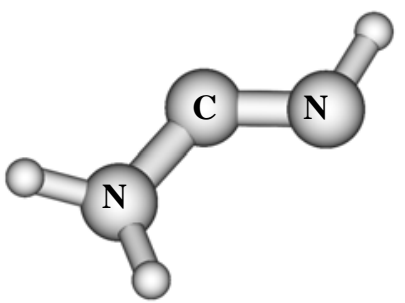

$E_{\text {tot }}(\mathrm{ZPE}):-149.302201$ иа

H (ZPE): -149.297164 иа

G (ZPE): -149.327301 иа

S: $63.43 \mathrm{cal} / \mathrm{mol} / \mathrm{K}$ 
$\mathrm{Me}_{2} \mathrm{~N}+6^{*} a$ in the same calculation at important distance ( $\sim 10 \AA$, triplet state):

$E_{\text {tot }}(\mathrm{ZPE})$ : -283.742332 ua (correction of the basis set effect)

H (ZPE): - 283.732541 ua (correction of the basis set effect)

G (ZPE): - 283.785964 ua (correction of the basis set effect)

S: $112.44 \mathrm{cal} / \mathrm{mol} / \mathrm{K}$ (correction of the basis set effect)

$\mathrm{Me}_{2} \mathrm{~N}+6^{*} a$ in separate calculations:

$E_{\text {tot }}(\mathrm{ZPE}):-283.742480$ ua $\left[=E^{\mathrm{Me2N}}{ }_{\text {tot }}(\mathrm{ZPE})+E^{6^{*} a}{ }_{\text {tot }}(\mathrm{ZPE})\right]$

$H(Z P E):-283.731674$ ua $\left[=H^{M e 2 N}(Z P E)+H^{6 * a}(Z P E)\right]$

$G(Z P E):-283.794722$ иа $\left[=G^{M e 2 N}(Z P E)+G^{6 * a}(Z P E)\right]$

$S: 132.70 \mathrm{cal} / \mathrm{mol} / \mathrm{K}\left[=S^{\mathrm{Me} 2 \mathrm{~N}}+S^{6^{*} a}\right]$

6*b:

$\begin{array}{rrrr}\mathrm{N} & 1.283773 & 0.007353 & 0.011238 \\ \mathrm{C} & 0.115229 & -0.400195 & 0.007697 \\ \mathrm{~N} & -1.138606 & 0.108173 & -0.053526 \\ \mathrm{H} & 1.503489 & 1.008821 & -0.044572 \\ \mathrm{H} & -1.914320 & -0.506580 & 0.136255 \\ \mathrm{H} & -1.296705 & 1.090252 & 0.158151\end{array}$

$E_{\text {tot }}(\mathrm{ZPE}):-149.292430 \mathrm{ua}$

H (ZPE): -149.287286 иа

G (ZPE): -149.317638 иа

S: $63.88 \mathrm{cal} / \mathrm{mol} / \mathrm{K}$

$\mathrm{Me}_{2} \mathrm{~N}+6^{*} \mathrm{~b}$ in the same calculation at important distance ( $10 \AA$, triplet state):

$E_{\text {tot }}(Z P E):-283.732649$ ua (correction of the basis set effect)

$H$ (ZPE): -283.724605 иа (correction of the basis set effect)

G (ZPE): -283.768710 ua (correction of the basis set effect)

$S: 92.83 \mathrm{cal} / \mathrm{mol} / \mathrm{K}$ (correction of the basis set effect)

$M e_{2} N+6 * b$ in separate calculations:

$E_{\text {tot }}(\mathrm{ZPE}):-283.732709$ ua $\left[=E^{\mathrm{Me2N}}{ }_{\text {tot }}(\mathrm{ZPE})+E^{6^{* b}}{ }_{\text {tot }}(\mathrm{ZPE})\right]$

$H$ (ZPE): -283.721796 иа $\left[=H^{M e 2 N}(Z P E)+H^{6^{* b}}(\mathrm{ZPE})\right]$

$G(Z P E):-283.785059$ иа $\left[=G^{M e 2 N}(Z P E)+G^{6 * b}(Z P E)\right]$

$S: 133.15 \mathrm{cal} / \mathrm{mol} / \mathrm{K}\left[=S^{\mathrm{Me} 2 \mathrm{~N}}+S^{6^{*} b}\right]$ 


$$
\begin{gathered}
\mathrm{Me}_{2} \mathrm{~N}-\mathrm{NH} \\
\stackrel{\mathrm{C}}{\mathrm{C}}-\mathrm{NMe}_{2} \\
2 \mathrm{Me}^{*}
\end{gathered}
$$

For the singlet carbene $\mathrm{Me}_{2} \mathrm{~N}(\mathrm{NH})-\mathrm{C}-\mathrm{NMe}_{2} 2 \mathbf{M e}^{*}$, four minima were found on the potential energy surface. The different isomers are drawn in Figure $\mathbf{S 4}$ and the geometrical parameters are presented in Table S3.
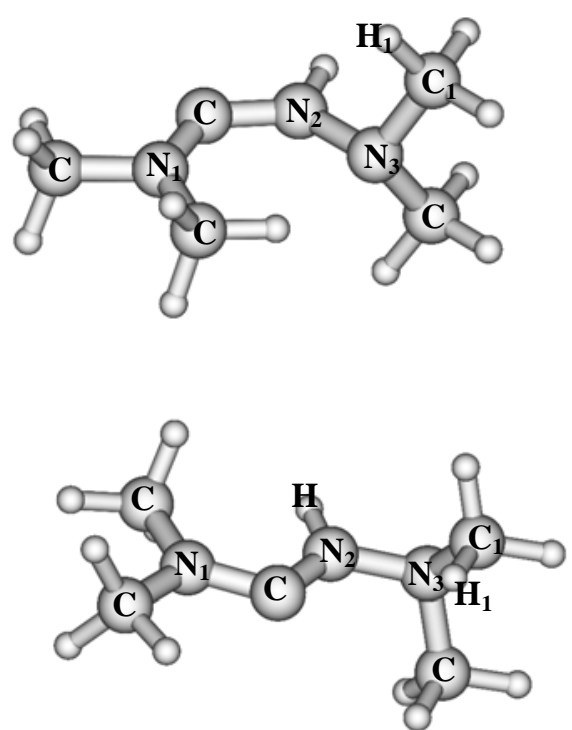
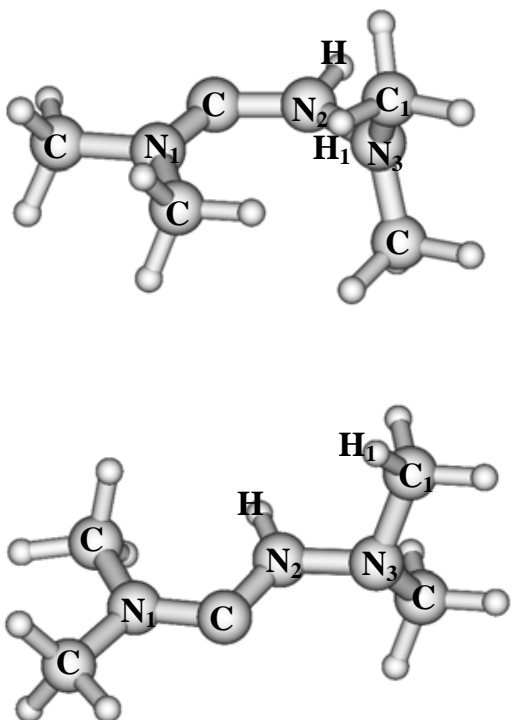

Figure S4: Different minima found on the potential energy surface for the singlet carbene $\mathrm{Me}_{2} \mathrm{~N}(\mathrm{NH})-\mathrm{C}-\mathrm{NMe}_{2}\left(2 \mathrm{Me}^{*}\right)$.

Table S3: For the different structures $2 \mathbf{M e}^{*} \mathbf{a}, 2 \mathbf{M e}^{*} \mathbf{b}, 2 \mathbf{M e}^{*} \mathbf{c}, 2 \mathbf{M e}^{*} \mathbf{d}, \mathbf{T S M e}_{\text {frag }}$ and $3 \mathbf{M e}$,

\begin{tabular}{|c|c|c|c|c|c|c|c|}
\hline & $2 *$ Mea & 2*Meb & 2*Mec & 2*Med & TSMe $_{\text {frag }}$ & 3Me & 4 \\
\hline $\mathrm{N}_{1} \mathrm{C}$ & 1.341 & 1.341 & 1.346 & 1.349 & 1.341 & 1.382 & $\mathbf{N}_{3} \mathbf{C}_{2}: 1.452$ \\
\hline $\mathbf{N}_{2} \mathrm{C}$ & 1.345 & 1.350 & 1.338 & 1.342 & 1.248 & 1.279 & $\mathbf{N}_{3} \mathbf{C}_{2}: 1.267$ \\
\hline $\mathbf{N}_{2} \mathbf{N}_{3}$ & 1.445 & 1.434 & 1.426 & 1.412 & 1.979 & l & / \\
\hline $\mathrm{C}_{1} \mathrm{H}_{1}$ & 3.435 & 3.575 & 3.247 & 3.216 & 1.251 & 1.094 & I \\
\hline $\mathrm{N}_{1} \mathrm{CN}_{2}$ & 121.54 & 123.00 & 114.61 & 113.65 & 132.83 & 130.31 & 117.71 \\
\hline $\mathrm{CN}_{2} \mathrm{~N}_{3}$ & 132.90 & 138.46 & 121.73 & 120.16 & 135.38 & 111.99 & / \\
\hline$\Sigma \mathbf{N}_{1}$ & 360.0 & 360.0 & 360.0 & 360.0 & 359.4 & 356.18 & I \\
\hline$\Sigma \mathbf{N}_{2}$ & 360.0 & 360.0 & 360.0 & 359.9 & l & l & I \\
\hline$\Sigma \mathbf{N}_{3}$ & 330.54 & 353.15 & 333.06 & 333.18 & l & l & I \\
\hline$\Delta \mathbf{E}$ & 2.4 & 11.0 & 0 & 2.08 & 24.6 & 3Me & $4:-31.2^{a}$ \\
\hline
\end{tabular}
all distances are given in angstroms $(\AA)$, the bond angles in degrees $\left(^{\circ}\right)$ and the energy differences in $\mathrm{kcal} / \mathrm{mol}$.

${ }^{\mathrm{a}}$ calculated from the energies of the compounds 3Me and 4 separately optimized. - $34.2 \mathrm{kcal} / \mathrm{mol}$ when the compounds $\mathbf{3}$ and $\mathbf{4}$ are carried out in the same calculation (correction of the basis set effect). 
For the $\beta$-fragmentation process of $\mathbf{2} \mathbf{M e}$, a cyclic transition state $\mathbf{T S M} \mathbf{e}_{\mathbf{f r a g}}$ has been located on the potential energy surface. The latter $\left(\mathbf{2} \mathbf{M e} \mathbf{e}^{*} \mathbf{c}\right)$ as well as the connected minima (3Me and 4) are drawn in Figure S5. The geometrical parameters of $2 \mathbf{M e}^{*} \mathbf{c}, \mathbf{T S M e}_{\text {frag }}, \mathbf{3 M e}$ and $\mathbf{4}$ are presented in Table S3. The rearrangement was predicted to be highly exothermic [ $-31.2 \mathrm{kcal} / \mathrm{mol}(\Delta \mathrm{E})$ or $\sim-42.0 \mathrm{kcal} / \mathrm{mol}(\Delta \mathrm{G})]$ and the cyclic transition state has been located $24.6 \mathrm{kcal} / \mathrm{mol}(\Delta \mathrm{E})$ or $25.5 \mathrm{kcal} / \mathrm{mol}(\Delta \mathrm{G})$ higher in energy than the most stable conformer of 2Me* $(\mathbf{2 M e *} \mathbf{c})$. These different values are close to those obtained for the $\beta$-fragmentation process of $2^{*}$ and show that the entropic effects are also weak in this case.

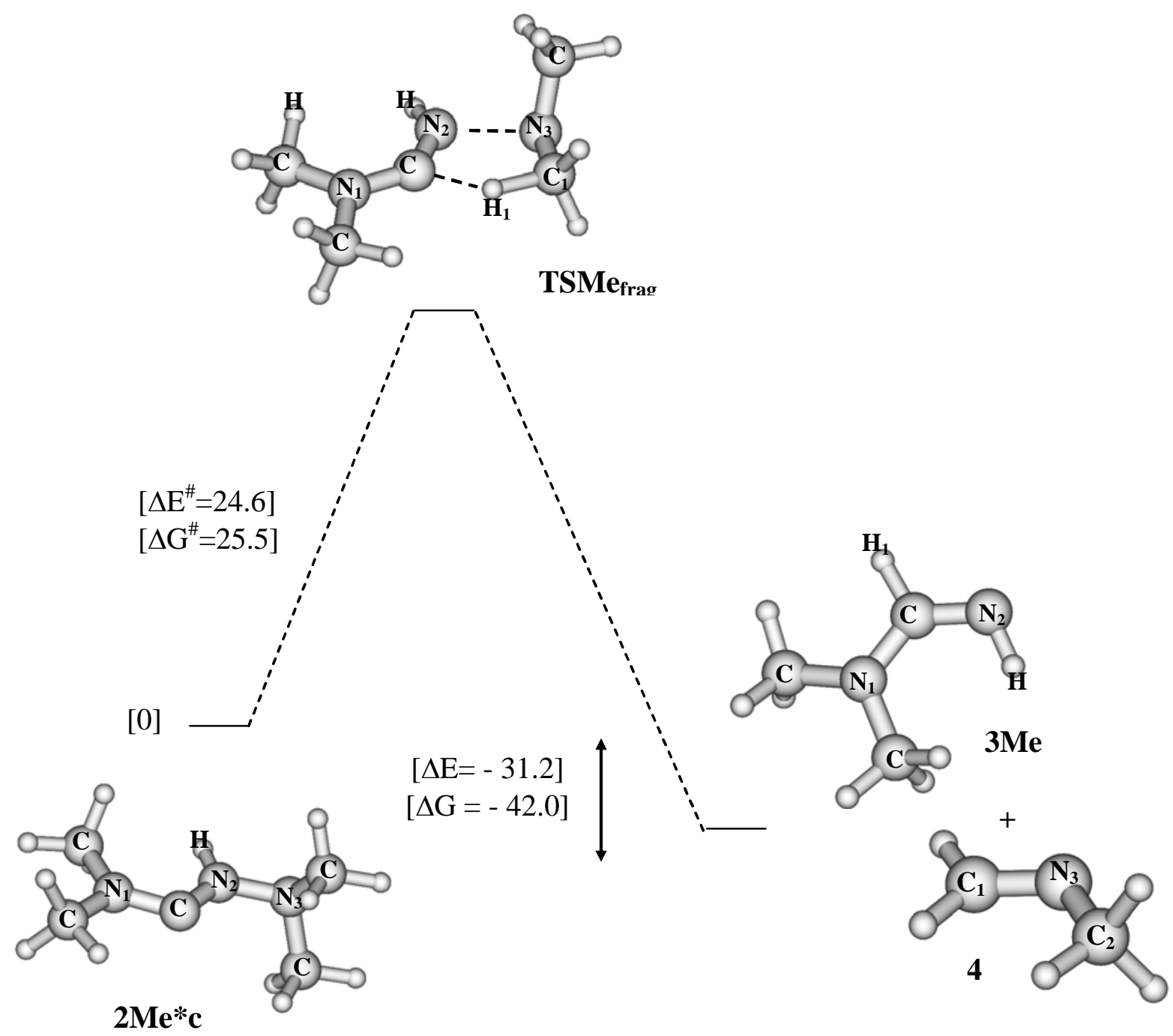

Figure S5: Transition state of the $\beta$-fragmentation reaction and minima connected to the latter. Energy differences in $\mathrm{kcal} / \mathrm{mol}$. 
For the radical $\mathbf{6 M e}$, 2 minima were found on the potential energy surface. The 2 structures are drawn in Figure S6. The geometrical parameters and the energy differences are presented in Table S4.
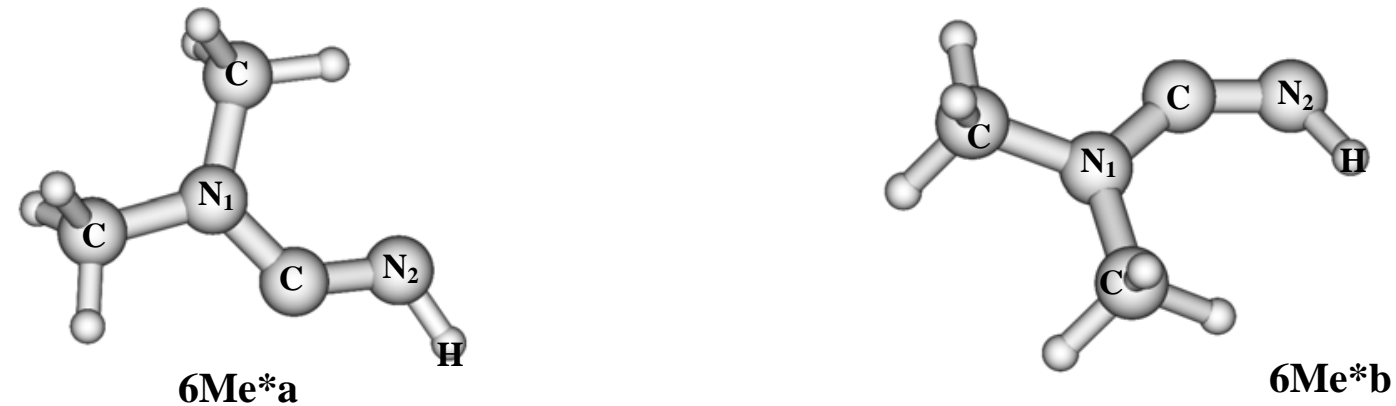

Figure S6: trans and cis isomers for the radical $\mathbf{6 M e *}$.

Table S2: For the two structures $6 \mathbf{M e}^{*} \mathbf{a}, \mathbf{6 M e} * \mathbf{b}$ all distances are given in angstroms $(\AA)$, the bond angles in degrees $\left(^{\circ}\right)$ and the energy differences in $\mathrm{kcal} / \mathrm{mol}$.

\begin{tabular}{ccc}
\hline & $\mathbf{6 M e} \mathbf{*}^{\mathbf{a}}$ & $\mathbf{6 M e * b}$ \\
\hline $\mathbf{N}_{\mathbf{1}} \mathbf{C}$ & 1.345 & 1.349 \\
$\mathbf{N}_{\mathbf{2}} \mathbf{C}$ & 1.260 & 1.242 \\
$\mathbf{N}_{\mathbf{1}} \mathbf{C N}_{\mathbf{2}}$ & 129.30 & 139.13 \\
$\boldsymbol{\Sigma}_{\mathbf{1}}$ & 359.4 & 359.0 \\
$\Delta \mathbf{E}$ & 0 & 6.8 \\
\hline
\end{tabular}

The fragmentation of the singlet carbene $\mathbf{2} \mathbf{M e} *$ can lead to the two radicals $\mathbf{M e}_{2} \mathbf{N}$ and 6Me*. The $\mathrm{N}-\mathrm{N}$ bond energy of $\mathbf{2} \mathbf{M e}^{*}$ has been estimated to be the energy required for homolytic fragmentation of $\mathbf{2} \mathbf{M e} *$. Accordingly, the N-N energy of $\mathbf{2} \mathbf{M e *}$ a was estimated at $21.2 \mathrm{kcal} / \mathrm{mol}\left(\mathrm{E}_{6 \mathbf{6 m}} \mathbf{*}_{\mathbf{a}}+\mathrm{E}_{\mathbf{M e} 2 \mathbf{N}}-\mathrm{E}_{2 \mathbf{M e}^{*} \mathbf{a}}\right)$ or $7.0 \mathrm{kcal} / \mathrm{mol}$ (free energy: $\mathrm{G}_{6 \mathbf{M e}} \mathbf{*}_{\mathbf{a}}+\mathrm{G}_{\mathbf{M e 2 N}}-$

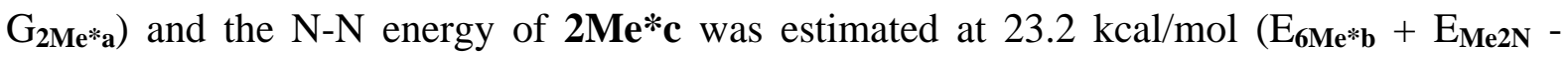

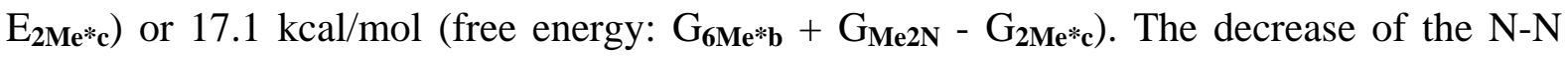
energy of $\mathbf{2} \mathbf{M e *}$, when taking into account the free energy, shows that the entropic factors are in favor of the dissociative radical pathway. These different values are close to those obtained for the radical fragmentation of $2 *$. 
Cartesian coordinates for all the compounds, Zero point Energy, Enthalpy, free energy in ua and entropy in $\mathrm{cal} / \mathrm{mol} / \mathrm{K}$ :

\section{2*Mea:}

$\begin{array}{lrr}\mathrm{N} & 0.541205 & -0.961423 \\ \mathrm{C} & -0.796895 & -1.094406 \\ \mathrm{~N} & -1.608167 & -0.026464 \\ \mathrm{~N} & 1.415651 & 0.189457 \\ \mathrm{H} & 1.047263 & -1.842706 \\ \mathrm{C} & -3.040554 & -0.309390 \\ \mathrm{C} & -1.317589 & 1.416119 \\ \mathrm{C} & 2.216954 & 0.203114 \\ \mathrm{C} & 2.245353 & 0.144163 \\ \mathrm{H} & 2.903330 & 1.018588 \\ \mathrm{H} & 2.877585 & -0.762019 \\ \mathrm{H} & 1.603192 & 0.166639 \\ \mathrm{H} & 2.875925 & 1.076992 \\ \mathrm{H} & 1.554266 & 0.269705 \\ \mathrm{H} & 2.845441 & -0.700161 \\ \mathrm{H} & -1.761897 & 1.905613 \\ \mathrm{H} & -0.243276 & 1.575800 \\ \mathrm{H} & -1.763901 & 1.869810 \\ \mathrm{H} & -3.522457 & 0.140306 \\ \mathrm{H} & -3.526701 & 0.102514 \\ \mathrm{H} & -3.173202 & -1.389673\end{array}$
$0.010810-0.372594$
$0.017595 \quad 0.146841$
$-0.002245-0.391656$
$-0.006111-0.312960$
$0.032059 \quad 0.253745$
$0.006907-0.141321$
$-0.031998-0.191796$
$1.215928-0.148348$
$-1.207823-0.148727$
$-1.227068 \quad 0.109785$
$-1.2579350 .080823$
- $2.090985 \quad 0.125736$
$1.207321 \quad 0.109661$
$2.081551 \quad 0.125504$
$1.325687 \quad 0.081174$
$0.844985 \quad 0.101180$
$-0.037490 \quad 0.151300$
$-0.9270320 .101369$
$0.886260 \quad 0.095570$
$-0.8884820 .095714$
$0.030300 \quad 0.128826$

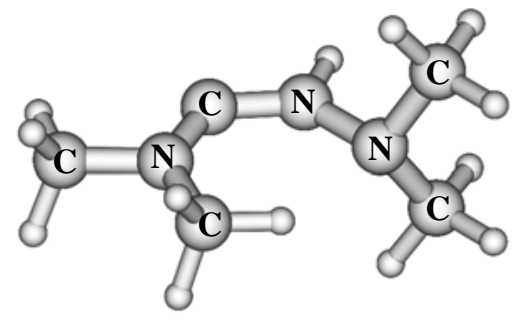

$E_{\text {tot }}$ (ZPE): -362.341884 ua (B3LYP), $E_{\text {tot }}$ (ZPE): - 362.341887 uа (UB3LYP)

H (ZPE): -362.330667 ua, G (ZPE): -362.377120 ua, S: $97.77 \mathrm{cal} / \mathrm{mol} / \mathrm{K}$

\section{2*Meb:}

$\begin{array}{lr}\text { N } & -0.544577 \\ \mathrm{C} & 0.804832 \\ \mathrm{~N} & 1.491881 \\ \mathrm{~N} & -1.653522 \\ \mathrm{H} & -0.889101 \\ \mathrm{C} & 2.948089 \\ \mathrm{C} & 1.058710 \\ \mathrm{C} & -1.956691 \\ \mathrm{C} & -1.959900 \\ \mathrm{H} & -3.017517 \\ \mathrm{H} & -1.369835 \\ \mathrm{H} & -1.804178 \\ \mathrm{H} & -3.014016 \\ \mathrm{H} & -1.800167 \\ \mathrm{H} & -1.365195 \\ \mathrm{H} & 1.450965 \\ \mathrm{H} & 1.443360 \\ \mathrm{H} & -0.020051 \\ \mathrm{H} & 3.381739 \\ \mathrm{H} & 3.381164 \\ \mathrm{H} & 3.196108\end{array}$

-1.215164
-1.163596
-0.011420
-0.305969
-2.163659
-0.135211
1.389961
0.367157
0.362653
0.654198
1.271287
-0.339126
0.659764
-0.332072
1.275807
1.908509
1.908937
1.461875
0.343961
0.347470
-1.194856

$\begin{array}{rr}0.0 \odot 1264 & -0.371984 \\ 0.000911 & 0.146907 \\ -0.0 \odot \odot 535 & -0.392265 \\ 0.000942 & -0.333107 \\ 0.0 \odot 2523 & 0.280903 \\ 0.000151 & -0.142981 \\ -0.002342 & -0.173429 \\ 1.247984 & -0.144613 \\ -1.247720 & -0.144613 \\ -1.243294 & 0.106608 \\ -1.462713 & 0.079536 \\ -2.071066 & 0.119045 \\ 1.244642 & 0.106566 \\ 2.073353 & 0.119013 \\ 1.458675 & 0.079579 \\ -0.887171 & 0.109059 \\ 0.885612 & 0.109128 \\ -0.0 \odot 7175 & 0.119064 \\ -0.888588 & 0.097366 \\ 0.887270 & 0.097327 \\ 0.002341 & 0.132826\end{array}$

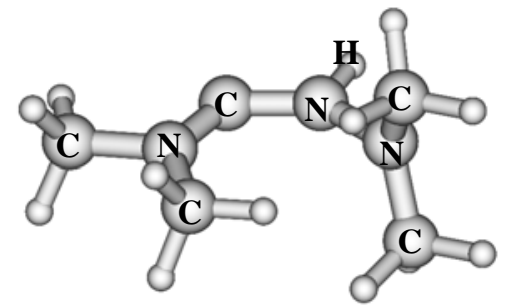

$E_{\text {tot }}(\mathrm{ZPE}):-362.328145$ ua

H (ZPE): -362.316614 иа, G (ZPE): -362.363621 иа, S: $98.93 \mathrm{cal} / \mathrm{mol} / \mathrm{K}$ 


\section{2*Mec:}

$\begin{array}{rrrrr}\mathrm{N} & -0.466452 & 0.594128 & -0.015078 & -0.383618 \\ \mathrm{C} & 0.373904 & -0.447221 & 0.011653 & 0.179822 \\ \mathrm{~N} & 1.678174 & -0.115151 & 0.009786 & -0.399580 \\ \mathrm{~N} & -1.881857 & 0.416439 & -0.009407 & -0.303449 \\ \mathrm{H} & -0.221916 & 1.581776 & -0.044004 & 0.232891 \\ \mathrm{C} & 2.666274 & -1.186296 & 0.014581 & -0.149895 \\ \mathrm{C} & 2.201153 & 1.254758 & -0.019283 & -0.198986 \\ \mathrm{C} & -2.311978 & -0.306060 & -1.208396 & -0.145216 \\ \mathrm{C} & -2.309997 & -0.253234 & 1.220428 & -0.145419 \\ \mathrm{H} & -3.402664 & -0.315597 & 1.219893 & 0.098597 \\ \mathrm{H} & -1.886670 & -1.263101 & 1.322774 & 0.109573 \\ \mathrm{H} & -1.998935 & 0.345919 & 2.080790 & 0.101258 \\ \mathrm{H} & -3.404383 & -0.372282 & -1.201400 & 0.098627 \\ \mathrm{H} & -2.006242 & 0.257079 & -2.094662 & 0.101121 \\ \mathrm{H} & -1.884885 & -1.317712 & -1.268900 & 0.109517 \\ \mathrm{H} & 1.929821 & 1.769990 & -0.950265 & 0.120336 \\ \mathrm{H} & 1.817927 & 1.842206 & 0.824828 & 0.119211 \\ \mathrm{H} & 3.289931 & 1.238523 & 0.051997 & 0.118140 \\ \mathrm{H} & 3.296308 & -1.154610 & -0.884591 & 0.101485 \\ \mathrm{H} & 3.322015 & -1.120774 & 0.893121 & 0.100998 \\ \mathrm{H} & 2.124511 & -2.131001 & 0.039418 & 0.134506\end{array}$

$E_{\text {tot }}$ (ZPE): -362.345682 ua, $E_{\text {tot }}$ (ZPE): -362.345681 иа (UB3LYP)

H (ZPE): -362.334190 иа, G (ZPE): -362.382330 иа, S: $101.32 \mathrm{cal} / \mathrm{mol} / \mathrm{K}$

\section{2*Med:}

$\begin{array}{lrrrr}\mathrm{N} & -0.464660 & 0.127131 & 0.014577 & -0.378036 \\ \mathrm{C} & 0.589005 & -0.643562 & -0.298150 & 0.183282 \\ \mathrm{~N} & 1.779349 & -0.068070 & -0.029483 & -0.399242 \\ \mathrm{~N} & -1.778011 & -0.354839 & -0.177421 & -0.256230 \\ \mathrm{H} & -0.423520 & 1.058966 & 0.447545 & 0.204394 \\ \mathrm{C} & 2.986309 & -0.818058 & -0.352705 & -0.148939 \\ \mathrm{C} & 1.981132 & 1.266225 & 0.545939 & -0.198097 \\ \mathrm{C} & -2.435236 & -0.554168 & 1.108779 & -0.151869 \\ \mathrm{C} & -2.514977 & 0.542187 & -1.057957 & -0.153390 \\ \mathrm{H} & -3.516546 & 0.135811 & -1.226089 & 0.111277 \\ \mathrm{H} & -2.626261 & 1.565727 & -0.648393 & 0.068565 \\ \mathrm{H} & -1.996464 & 0.605687 & -2.017267 & 0.125895 \\ \mathrm{H} & -3.435405 & -0.964354 & 0.940469 & 0.110439 \\ \mathrm{H} & -1.856088 & -1.271431 & 1.694529 & 0.126506 \\ \mathrm{H} & -2.541312 & 0.378991 & 1.696120 & 0.068356 \\ \mathrm{H} & 3.047581 & 1.454879 & 0.680586 & 0.117327 \\ \mathrm{H} & 1.578974 & 2.051626 & -0.107601 & 0.118427 \\ \mathrm{H} & 1.498956 & 1.354571 & 1.528345 & 0.116361 \\ \mathrm{H} & 3.601605 & -0.288840 & -1.093026 & 0.099422 \\ \mathrm{H} & 3.601384 & -0.990704 & 0.540818 & 0.098838 \\ \mathrm{H} & 2.672945 & -1.776233 & -0.765182 & 0.136596\end{array}$

$E_{\text {tot }}(\mathrm{ZPE}):-362.342371$ иа

H (ZPE): -362.330913 иа, G (ZPE): -362.379014 ua, S: $98.93 \mathrm{cal} / \mathrm{mol} / \mathrm{K}$ 


\section{TSMe $_{\text {frag: }}$}

$\begin{array}{lrrrr}\mathrm{C} & -2.904451 & -0.683793 & 0.544491 & -0.135847 \\ \mathrm{~N} & -2.200539 & 0.034330 & -0.505526 & -0.306727 \\ \mathrm{C} & -1.875223 & 1.333731 & -0.188008 & -0.177697 \\ \mathrm{~N} & -0.424935 & -0.811146 & -0.287928 & -0.490854 \\ \mathrm{C} & 0.432233 & 0.067343 & -0.062586 & 0.376254 \\ \mathrm{~N} & 1.762787 & 0.023155 & 0.095598 & -0.422680 \\ \mathrm{H} & -0.410951 & -1.750634 & -0.656024 & 0.244419 \\ \mathrm{H} & -1.964655 & 2.031182 & -1.026680 & 0.100101 \\ \mathrm{H} & -2.274981 & 1.740765 & 0.751034 & 0.076207 \\ \mathrm{H} & -0.642463 & 1.248808 & 0.008562 & 0.093073 \\ \mathrm{H} & -3.975761 & -0.452715 & 0.463765 & 0.105553 \\ \mathrm{H} & -2.792037 & -1.764678 & 0.405496 & 0.086883 \\ \mathrm{H} & -2.580824 & -0.427407 & 1.567130 & 0.081648 \\ \mathrm{C} & 2.529305 & -1.211537 & -0.051167 & -0.174618 \\ \mathrm{C} & 2.533968 & 1.250917 & 0.227446 & -0.162035 \\ \mathrm{H} & 3.373228 & -1.213137 & 0.647274 & 0.115470 \\ \mathrm{H} & 1.889664 & -2.065558 & 0.180652 & 0.117200 \\ \mathrm{H} & 2.926835 & -1.331308 & -1.068701 & 0.119164 \\ \mathrm{H} & 3.207895 & 1.194090 & 1.091522 & 0.107966 \\ \mathrm{H} & 3.143152 & 1.442618 & -0.666870 & 0.110104 \\ \mathrm{H} & 1.844712 & 2.083640 & 0.366782 & 0.136615\end{array}$

frequency: $-694.53 \mathrm{~cm}^{-1}$

$E_{\text {tot }}(\mathrm{ZPE}):-362.306462$ ua

H (ZPE): -362.295232 иа, G (ZPE): -362.341634 ua, S: $97.66 \mathrm{cal} / \mathrm{mol} / \mathrm{K}$

\section{Me:}

$\begin{array}{rrrrr}\mathrm{C} & 0.836535 & -0.677680 & -0.060788 & 0.278661 \\ \mathrm{~N} & 2.022835 & -0.217293 & 0.071415 & -0.546497 \\ \mathrm{H} & 2.029874 & 0.800924 & 0.170032 & 0.208792 \\ \mathrm{H} & 0.720128 & -1.762080 & -0.146353 & 0.092774 \\ \mathrm{~N} & -0.373019 & -0.017027 & -0.158046 & -0.387668 \\ \mathrm{C} & -1.608179 & -0.745122 & 0.076697 & -0.171396 \\ \mathrm{C} & -0.422110 & 1.423894 & 0.000891 & -0.181674 \\ \mathrm{H} & -2.397084 & -0.385130 & -0.593143 & 0.115724 \\ \mathrm{H} & -1.449873 & -1.807254 & -0.125529 & 0.122919 \\ \mathrm{H} & -1.967308 & -0.645170 & 1.112415 & 0.112244 \\ \mathrm{H} & -1.379239 & 1.794718 & -0.374988 & 0.115350 \\ \mathrm{H} & -0.315114 & 1.743467 & 1.049088 & 0.115934 \\ \mathrm{H} & 0.372429 & 1.894211 & -0.585903 & 0.124948\end{array}$
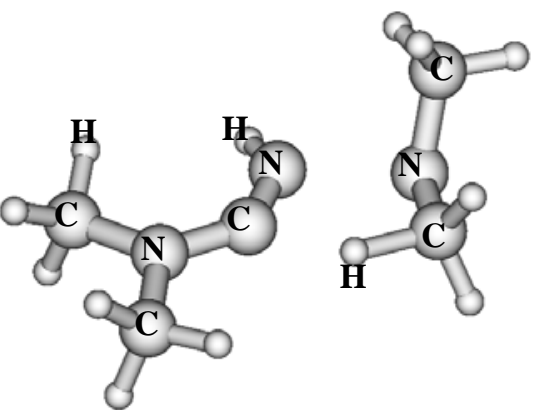

$E_{\text {tot }}(\mathrm{ZPE}):-228.514506$ ua

H (ZPE): -228.507227 ua, G (ZPE): -228.543854 ua, S: $77.09 \mathrm{cal} / \mathrm{mol} / \mathrm{K}$ 


\section{$3 M e+4$ in the same calculation at $\sim 3.7 \AA$ :}

$E_{\text {tot }}(\mathrm{ZPE}):-362.400245$ ua (correction of the basis set effect)

H (ZPE): - 362.387225 ua (correction of the basis set effect)

$G$ (ZPE): 362.440581 ua (correction of the basis set effect)

S: $112.30 \mathrm{cal} / \mathrm{mol} / \mathrm{K}$ (correction of the basis set effect)

\section{Me and 4 in separate calculations:}

$E_{\text {tot }}(\mathrm{ZPE}):-362.395272$ ua $\left[=E^{3 M e}{ }_{\text {tot }}(\mathrm{ZPE})+E_{\text {tot }}^{4}(\mathrm{ZPE})\right]$

$H$ (ZPE): -362.383190 иа $\left[=H^{3 M e}(Z P E)+H^{4}(Z P E)\right]$

$G(Z P E):-361.449348$ ua $\left[=G^{3 M e}(Z P E)+G^{4}(Z P E)\right]$

S: $139.24 \mathrm{cal} / \mathrm{mol} / \mathrm{K}\left[=S^{3 M e}+S^{4}\right]$

\section{6*Mea:}

$\begin{array}{rrrrr}\mathrm{N} & -2.003685 & -0.077350 & 0.045463 & -0.561467 \\ \mathrm{C} & -0.868856 & -0.619433 & -0.035161 & 0.312151 \\ \mathrm{~N} & 0.348152 & -0.047866 & -0.068429 & -0.398132 \\ \mathrm{H} & -2.812740 & -0.685684 & -0.038787 & 0.255591 \\ \mathrm{C} & 0.518348 & 1.402181 & 0.004887 & -0.173876 \\ \mathrm{C} & 1.543162 & -0.866463 & 0.027737 & -0.157852 \\ \mathrm{H} & 1.243082 & 1.734812 & -0.747554 & 0.110730 \\ \mathrm{H} & -0.445710 & 1.873859 & -0.183908 & 0.150991 \\ \mathrm{H} & 0.880149 & 1.711160 & 0.995087 & 0.113660 \\ \mathrm{H} & 2.248963 & -0.617609 & -0.774929 & 0.111078 \\ \mathrm{H} & 2.056345 & -0.720914 & 0.988990 & 0.110535 \\ \mathrm{H} & 1.262717 & -1.916815 & -0.062915 & 0.126595\end{array}$

$E_{\text {tot }}(Z P E):-227.867734$ ua

H (ZPE): -227.861037 иа, G (ZPE): -227.898593 иа, S (ZPE): $81.15 \mathrm{cal} / \mathrm{mol} / \mathrm{K}$

\section{$M e_{2} N+6^{*} M e a$ in the same calculation at important distance ( $10 \AA$, triplet state):}

$E_{\text {tot }}(Z P E)$ :-362.307709 ua (correction of the basis set effect)

$H$ (ZPE): -362.294417 ua (correction of the basis set effect)

G (ZPE): - 362.356327 ua (correction of the basis set effect)

$S: 130.30 \mathrm{cal} / \mathrm{mol} / \mathrm{K}$ (correction of the basis set effect)

\section{$M e_{2} N+6 M e^{*} a$ in separate calculations:}

$E_{\text {tot }}(\mathrm{ZPE}):-362.308013$ ua $\left[=E^{\mathrm{Me2N}}{ }_{\text {tot }}(\mathrm{ZPE})+E^{6^{*} a M e}{ }_{\text {tot }}(\mathrm{ZPE})\right]$

$H(Z P E):-362.295547$ ua $\left[=H^{M e 2 N}{ }_{t o t}(Z P E)+H^{6 * a M e}{ }_{\text {tot }}(Z P E)\right]$

$G(Z P E):-362.366014$ ua $\left[=G^{M e 2 N}(Z P E)+G^{6 * a M e}(Z P E)\right]$

$S: 150.42 \mathrm{cal} / \mathrm{mol} / \mathrm{K}\left[=S^{M e 2 N}+S^{6^{*} a M e}\right]$ 
6*Meb:

$\begin{array}{lrrrr}\mathrm{N} & -2.062659 & -0.310814 & 0.016384 & -0.547254 \\ \mathrm{C} & -0.864009 & -0.631581 & -0.048514 & 0.341784 \\ \mathrm{~N} & 0.349109 & -0.042551 & -0.085763 & -0.398040 \\ \mathrm{H} & -2.355476 & 0.647238 & 0.236361 & 0.224100 \\ \mathrm{C} & 0.495123 & 1.408511 & 0.002720 & -0.175765 \\ \mathrm{C} & 1.558446 & -0.840140 & 0.042925 & -0.159875 \\ \mathrm{H} & 1.300097 & 1.742602 & -0.660943 & 0.120377 \\ \mathrm{H} & -0.434334 & 1.884526 & -0.316780 & 0.118798 \\ \mathrm{H} & 0.729180 & 1.737619 & 1.024848 & 0.119016 \\ \mathrm{H} & 2.284740 & -0.566353 & -0.732280 & 0.112014 \\ \mathrm{H} & 2.034836 & -0.699684 & 1.023520 & 0.111502 \\ \mathrm{H} & 1.298448 & -1.893135 & -0.071853 & 0.133318\end{array}$

$E_{\text {tot }}(\mathrm{ZPE}):-227.856909$ ua

H: -227.849133 иа, G: -227.887627 ua, S: $81.02 \mathrm{cal} / \mathrm{mol} / \mathrm{K}$

$\mathrm{Me}_{2} \mathrm{~N}+6^{*} \mathrm{Meb}$ in the same calculation at important distance ( $10 \AA$, triplet state):

$E_{\text {tot }}(\mathrm{ZPE})$ : -362.296975 ua (correction of the basis set effect)

$H$ (ZPE): -362.285372 ua (correction of the basis set effect)

$G$ (ZPE ): -362.340643 ua (correction of the basis set effect)

S: $116.33 \mathrm{cal} / \mathrm{mol} / \mathrm{K}$ (correction of the basis set effect)

$\mathrm{Me}_{2} \mathrm{~N}+6^{*} \mathrm{Meb}$ in separate calculations:

$E_{\text {tot }}(\mathrm{ZPE}):-362.297188$ ua $\left[=E^{\mathrm{Me2N}}{ }_{\text {tot }}(\mathrm{ZPE})+E^{6^{*} \mathrm{Meb}}{ }_{\text {tot }}(\mathrm{ZPE})\right]$

$H$ (ZPE): -362.283643 иа $\left[=H^{M e 2 N}(Z P E)+H^{6 * M e b}(Z P E)\right]$

$G(\mathrm{ZPE}):-362.355048$ ua $\left[=G^{M e 2 N}(\mathrm{ZPE})+G^{6 * M e b}(\mathrm{ZPE})\right]$

$S: 150.29 \mathrm{cal} / \mathrm{mol} / \mathrm{K}\left[=S^{M e 2 N}+S^{6^{*} M e}\right]$

\section{Acknowledgement:}

We thank Dr Gijs Schaftenaar for allowing us to use his graphic program molden. ${ }^{\text {S8 }}$ We also thank the Institut du Developpement de Ressources en Informatique Scientifique (IDRIS, Orsay, France), administered by the CNRS, for the calculation facilities. 


\section{References:}

(S1) Wieland, G.; Simchen, G. Liebigs Ann. Chem. 1985, 11, 2178-2193.

(S2) Peterson, L. K.; Thé, K. I. Can. J. Chem. 1972, 50, 553-561.

(S3) Gaussian 98, M. J. Frisch, G. W. Trucks, H. B. Schlegel, G. E. Scuseria, M. A. Robb, J. R. Cheeseman, V. G. Zakrzewski, J. A. Montgomery, R. E. Stratman J. C. Burant S. Dapprich, J. M. Millam, A. D. Daniels, K. N. Kudin, M. C. Strain, O. Farkas, J. Tomasi, V. Barone, M. Cossi, R. Cammi, B. Mennucci, C. Pomelli, C. Adamo, S. Clifford, J. Ochterski, G. A. Petersson, P. Y. Ayala, Q. Cui, K. Morokuma, D. K. Malick, A. D. K. Rabuck, Raghavachari, J. B. Foresman, J. Cioslowswi, J. V. Ortiz, A. G. Baboul, B. B. Stefanov, G. Liu, A. Liashenko, P. Piskorz, I. Komaromi, R. Gomperts, R. Martin, D. J. Fox, T. Keith, M. A. Al-Laham, C. Y. Peng, A. Nanayakkara, C. Gonzalez, M. Challacombe, P. M. W. Gill, B. Jonhson, W. Chen, M. W. Wong, J. L. Andres, M. Head-Gordon, E. S. Replogle, J. A. Pople, Gaussian 98, Revision A.7, Gaussian, Inc., Pittsburgh PA, 1998.

(S4) W. J. Hehre, L. Radom, P.v.R. Schleyer, J. A. Pople, Ab Initio Molecular Orbital Theory, John Wiley and Sons: New York., 1986.

(S5) R. G. Parr, W. Yang, Functional Theory of Atoms and Molecules, R. Breslow, J. B. Goodenough, Eds., Oxford University Press: New York, 1989.

(S6) (a) A. D. Becke, Phys. Rev. 1988, A38, 3098-3100. (b) A. D. Becke, J. Chem. Phys. 1993, 98, 5648-5652. (c) C. Lee, W. Yang, R. G. Parr, Phys. Rev. 1988, B37, 785-789.

(S7) R. W. Alder, M. E. Blake, J. M. Oliva J. Phys. Chem. 1999, 103, 11200-11211.

(S8) G. Schaftenaar, J. H. Noordik J. Comput. - Aided Mol. Des. 2000, 14, 123-134. 